ISAHP 2003, Bali, Indonesia, August 7-9, 2003

\title{
TIME DEPENDENT DECISION-MAKING; DYNAMIC PRIORITIES IN AHP/ANP GENERALIZING FROM POINTS TO FUNCTIONS AND FROM REAL TO COMPLEX VARIABLES
}

\author{
Thomas L. Saaty \\ University of Pittsburgh \\ Pittsburgh, PA 15260 \\ saaty@katz.pitt.edu
}

\begin{abstract}
Keywords: dynamic priorities, time-dependent AHP, AHP, ANP, priority functions, time horizons, neural firing

Summary: The subject of time-dependent priorities is a new and potentially useful development in the AHP/ANP literature. It involves making paired comparisons not only of relative dominance of magnitudes as a starting point, but also of rates of change. Dealing with the future of human interaction and decisionmaking is very uncertain and confounding subject. We need caution in developing the subject if it is to work out to our advantage. This paper is mainly concerned with the theory of the subject. Examples are given to illustrate the method and detailed discussion is provided about how to derive time dependent priorities analytically for matrices of order up to four and numerically for matrices of all order. A brief representation and synthesis of time dependent neural firing and its hypermatrix is given.
\end{abstract}

\section{Introduction}

The Analytic Hierarchy Process (AHP) for decision-making is a theory of relative measurement based on paired comparisons used to derive absolute scales in the form of normalized ratio scales whose elements are then used as priorities. Matrices of pairwise comparisons are formed either by providing judgments to estimate dominance using absolute numbers from the 1 to 9 fundamental scale of the AHP, or by directly constructing the pairwise dominance ratios using actual measurements. The AHP can be applied to both tangible and intangible criteria based on the judgments of knowledgeable and expert people, although how to get measures for intangibles is its main concern. The weighting and adding synthesis process applied in the hierarchical structure of the AHP combines multidimensional scales of measurement into a single "uni-dimensional" scale of priorities. In the end we must fit our entire world experience into our system of priorities if we are going to understand it.

We are one-dimensional because we deal with the world separately for each dimension. Our failure to deal with the future with greater certainty and success may be attributable to two factors: 1) Lack of a comprehensive structure to represent any decision in the context of the anticipated environment that influences that future, and 2) Lack of an effective method to provide knowledge and judgments (certain or uncertain) within the structure and synthesize to capture the outcome that is a likely result of all the interactions of the factors included within the structure. Both these factors are essential for making sound decisions. Since prediction is made over time, it would be advantageous to determine the evolution of influences over different time horizons.

So far most of us have had no way to combine dollars with yards or pounds to trade them off. We would be truly multi-dimensional if we could combine the different dimensions into a single dimension that represents our priority of importance. That is precisely what the AHP and ANP help us do in a more or less precise way, depending on the level of experience that we bring to bear on a decision problem. Until recently, the AHP and

Proceedings $-7^{\text {th }}$ ISAHP 2003 Bali, Indonesia 
ANP have been static in that they have used numbers and derived numbers to represent priorities. What we need is to make them dynamic by using numbers or functions and then deriving either numbers that represent functions like expected values, or deriving functions directly to represent priorities over time. My aim here is to extend the AHP/ANP to deal with time dependent priorities; we may refer to them as DHP/DNP (Dynamic Hierarchy Process/Dynamic Network Process). At this point we may not know enough to develop the necessary fundamental scale of functions to use in making paired comparisons of intangibles. But if nothing else DHP and DNP work with tangibles now and we need to weight and trade off these tangibles as functions of time.

Time dependent decision-making that we call dynamic decision-making is a subject that we need today. It is multidimensional in space by including the important criteria and also in time. So far we have thought of our decisions as known alternatives to choose from. But these alternatives may evolve over time along with our preferences for them like stocks in the stock market whose prices constantly change over time. Our actions need to vary over time like a medicine capsule that releases different amounts of chemical at different times. Time dependent decisions are a reality and not a complicated idea that we can ignore. At a minimum they are needed in technical design problems in which the influences of several tangible design factors change over time and tradeoffs must be made among them to enable the system to respond differently and continuously over the time of its operation. But the power and potential of the subject lie in its use of judgment to make comparisons to derive relative real valued functions for intangibles from paired comparisons. Because we can do that for real numbers we can also do it for complex numbers. They have a modulus (magnitude) and an argument (direction), each of which is real. That is where we need to go later to derive relative complex functions from paired comparison expert judgments. The modulus is estimated in the usual way of comparing magnitudes and the argument or angle is estimated along the lines of rate of change comparison given below. In this manner the two parts of a complex number are derived from paired comparisons.

There are two ways to study dynamic decisions: structural, by including scenarios and time periods as elements in the structure that represents a decision, and functional by explicitly involving time in the judgment process. A third way would be a hybrid of these two.

The structural method is most familiar today and it involves using scenarios or time periods as factors in the hierarchic or network structure of a decision, and then making appropriate judgments. Generally contrast scenarios such as optimistic, status quo and pessimistic, or more specific ones such as different values for the economy or the stock market are put near or at the top of a hierarchy. The likelihood of the scenarios is first determined in terms of higher level criteria under the goal such as economic, political, social and technological, that are themselves prioritized according to their prevailing influences over a certain time horizon. Judgments are provided for the behavior of the alternatives with respect to the factors encountered under each scenario (see Wind and Saaty). Synthesis reveals the best alternative to follow in view of the mix of potential scenarios. For more detail about this method see my book on Analytical Planning where contrast scenarios are discussed. The other structural method is to put actual time periods at the "bottom" of the structure, prioritize them and finally combine their priorities by, for example, using the idea of expected value. This method was used in estimating when the US economy would recover and is illustrated in the next section.

The second approach where the judgments themselves change with time is functional in the literal sense. Whatever the structure may be, time dependent judgments are provided using functions from which priorities are then obtained and synthesized as one generally does with the AHP/ANP. We have two problems to solve when using dynamic judgments in the AHP/ANP. The first is what scale to use to represent dynamic judgments and how in this case it can be made to satisfy the axiom of homogeneity. The second is how to generate the principal eigenvector of a matrix whose order is more than four. Because 7 is an upper bound on producing results with reliable consistency, it is sufficient to find ways to derive the principal eigenvector of a matrix of order seven or less. This task can also be done in two ways, analytically and numerically. This paper is about both these ways but particularly about the mathematics of the functional way. In making pairwise comparisons we estimate how many times one element is more important than another with respect to a common property by forming their ratios if we know their

Proceedings $-7^{\text {th }}$ ISAHP 2003 Bali, Indonesia 
measurements and get an expert to tell us when we do not have their exact measurements. Each judgment is expressed with a number.

Suppose now that the measurements are a function of time. If we know the functions, we can form their ratios, but if we do not, what kind of typical functions can we use to represent ratios in a standard way just as we use numbers in a standard way. If humans have an intrinsic ability to look at the future of two stocks whose values appreciate over time and can say that on the whole one would be a better investment than the other, then one would like to capture this intuitive understanding by some standard functions to apply in all situations where we have to deal with intangibles over time. We have to do this kind of thinking even if we do not know much about the exact future. Like the US building a 60 billion dollar anti-missile system based on imagined threats over a future time horizon that may not materialize. The decision would be a function of time going forward or pulling back as the need may be. What are typical functions to use in describing in an uncertain way the ratio of anticipated variations of two functions over time? I will discuss and illustrate my proposed approach to this problem.

\section{A Structural Dynamics Example}

Let us consider the problem of the turn around of the US economy and introduce 3, 6, 12, 24 month time periods at the bottom (see Blair et. al). Decomposing the problem hierarchically, the top level consists of the primary factors that represent the forces or major influences driving the economy: "Aggregate Demand" factors, "Aggregate Supply" factors, and "Geopolitical Context." Each of these primary categories was then decomposed into subfactors represented in the second level. Under Aggregate Demand, we identified consumer spending, exports, business capital investment, shifts in consumer and business investment confidence, fiscal policy, monetary policy, and expectations with regard to such questions as the future course of inflation, monetary policy and fiscal policy. (We make a distinction between consumer and business investment confidence shifts and the formation of expectations regarding future economic developments.)

Under Aggregate Supply, we identified labor costs (driven by changes in such underlying factors as labor productivity and real wages), natural resource costs (e.g., energy costs), and expectations regarding such costs in the future. With regard to Geopolitical Context, we identified the likelihood of changes in major international political relationships and major international economic relationships as the principal subfactors. With regard to the subfactors under Aggregate Demand and Aggregate Supply, we recognized that they are, in some instances, interdependent. For example, a lowering of interest rates as the result of a monetary policy decision by the Federal Reserve should induce portfolio rebalancing throughout the economy. In turn, this should reduce the cost of capital to firms and stimulate investment, and simultaneously reduce financial costs to households and increase their disposable incomes. Any resulting increase in disposable income stimulates consumption and, at the margin, has a positive impact on employment and GNP. This assumes that the linkages of the economy are in place and are well understood. This is what the conventional macroeconomic conceptual models are designed to convey.

The third level of the hierarchy consists of the alternate time periods in which the resurgence might occur as of April 7, 2001: within three months, within six months, within twelve months, and within twenty-four months. Because the primary factors and associated subfactors are time-dependent, their relative importance had to be established in terms of each of the four alternative time periods. Thus, instead of establishing a single goal as one does for a conventional hierarchy, we used the bottom level time periods to compare the two factors at the top. This entailed creation of a feedback hierarchy known as a "holarchy" in which the priorities of the elements at the top level are determined in terms of the elements at the bottom level, thus creating an interactive loop. Figure 1 provides a schematic representation of the hierarchy we used to forecast the timing of the economic resurgence.

Proceedings $-7^{\text {th }}$ ISAHP 2003 Bali, Indonesia 


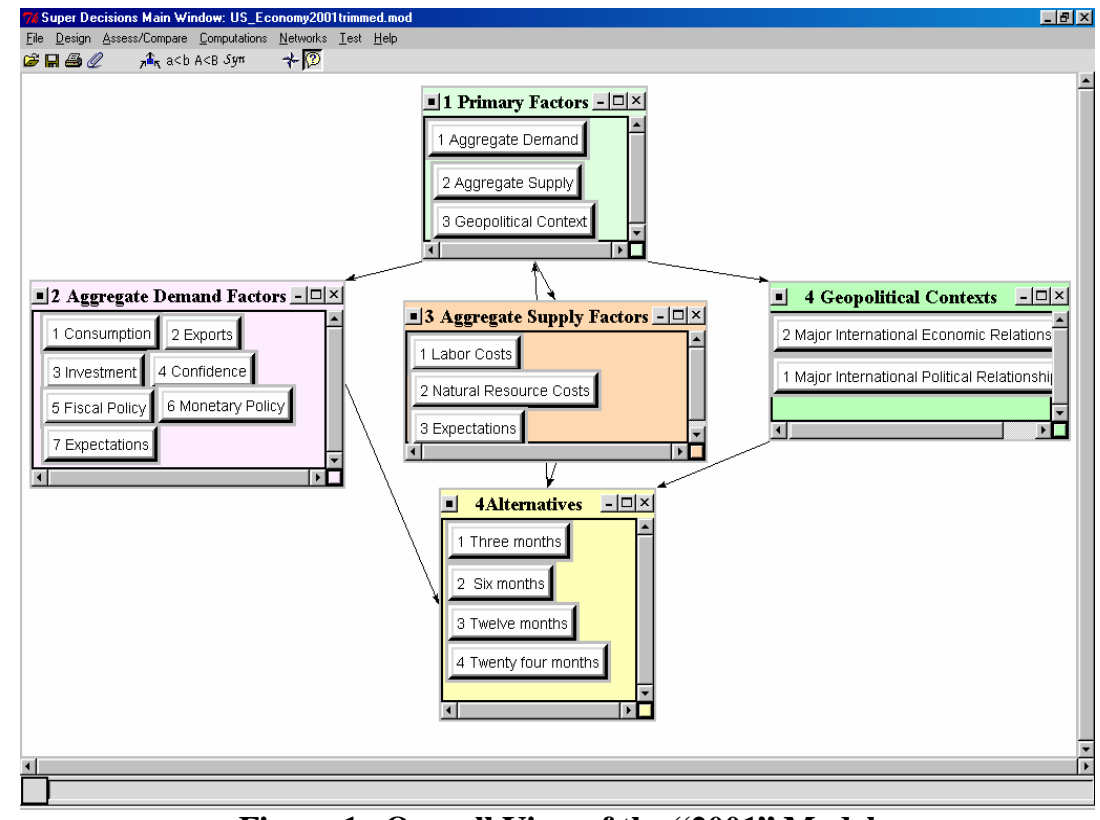

Figure 1 Overall View of the "2001" Model

To obtain our forecast, we subsequently multiplied each priority by the midpoint of its corresponding time interval and added the results (as one does when evaluating expected values):

\begin{tabular}{|c|c|c|c|}
\hline Time Period & $\begin{array}{l}\text { Midpoint of Time Period } \\
\text { xpressed in months from present, } \\
\text { with the current month as } 0 \text {.) }\end{array}$ & of Time Period & Midpoint $x$ Priority \\
\hline Three months & $0+(3-0) / 2=1.5$ & 0.30581 & 0.45871 \\
\hline Six months & $3+(6-3) / 2=4.5$ & 0.20583 & 0.92623 \\
\hline Twelve months 6 & $6+(12-6) / 2=9.0$ & 0.18181 & 1.63629 \\
\hline Twenty-four month & ths $\quad 12+(24-12) / 2=18.0$ & 0.30656 & OTAL $\frac{5.51808}{8.53932}$ \\
\hline
\end{tabular}

We interpret this to mean that the recovery would occur 8.54 months from the time of the forecasting exercise. The outcome of this paper had to be revised in view of the events of September 2001 and the threat of or actual war with Iraq at the time of this writing.

\section{Functional Dynamics: Numerical Solution of the Principal Eigenvalue Problem by Raising the Matrix to Powers - A Basic 3 x 3 Example}

Because priorities are obtained in the form of the principal eigenvector and because this vector is obtained by raising the paired comparisons matrix to powers, particularly in this case, we do not need to solve equations. We are fortunate in this special and rare case to do that. In the AHP one simply composes by multiplying and adding functions. So, in principle, there is no major theoretical difficulty in making decisions with dynamic judgments. In the ANP the problem is to obtain the limiting result of powers of the supermatrix with dynamic priorities. Because of its size, for the foreseeable future, the supermatrix would have to be solved numerically. It may even turn out in the long run that this is the more efficient way to obtain priorities from the supermatrix even when we have analytic expressions to represent priorities introduced in the usual way in the supermatrix to raise it to powers to obtain its limit for various values of time.

We remind the reader that if one has the principal eigenvector of a matrix, to test for consistency one obtains its principal eigenvalue by forming the scalar product of the principal eigenvector and the vector of 
column sums of the matrix. Generally, given an eigenvector, this is also the way to obtain its corresponding eigenvalue.

The typical form of a judgment matrix in dynamic form is:

$$
A(t)=\left[\begin{array}{cccc}
a_{11}(t) & a_{12}(t) & \cdots & a_{1 n}(t) \\
a_{21}(t) & a_{22}(t) & \cdots & a_{2 n}(t) \\
\vdots & \vdots & :: \vdots & \vdots \\
a_{n 1}(t) & a_{n 2}(t) & \cdots & a_{n n}(t)
\end{array}\right]
$$

$a_{i j}>0, a_{j i}(t)=a_{i j}^{-1}(t)$. As in the discrete case, when $A(t)$ is consistent, we have $a_{i j}(t)=w_{i}(t) / w_{j}(t)$.

The basic idea with the numerical approach is to obtain the time dependent principal eigenvector by simulation. One expresses the judgments functionally but then derives the eigenvector from the judgments for a fixed instant of time, substitutes the numerical values of the eigenvectors obtained for that instant in a supermatrix, solves the supermatrix problem and derives the priorities for the alternatives. Repeating the process for different values of time one generates a curve for the priorities of the alternatives and then approximates these values by curves with a functional form for each component of the eigenvector. It is sufficient to illustrate this entire procedure for one matrix.

Let us consider the 3 by 3 matrix with dynamic coefficients shown below. The rows of the two tables below the matrix give the principal eigenvector for the indicated values of $t$. They are then plotted in the diagram below that and an algorithm is used to find the analytic expressions for the best fitting curves for the three components of the eigenvector. This entire process can be made automatic in software for dynamic judgments.

$$
A(t)=\left[\begin{array}{ccc}
1 & a(t) & b(t) \\
1 / a(t) & 1 & c(t) \\
1 / b(t) & 1 / c(t) & 1
\end{array}\right] \begin{aligned}
& a(t)=0.1+t^{3} \\
& b(t)=1+2 t^{2} \\
& c(t)=1+\frac{1}{2} e^{t}
\end{aligned}
$$

Table 1 Eigenvectors for Different Values of Time for Matrix above

\begin{tabular}{|c|c|c|c|}
\hline $\mathrm{t}$ & $\mathrm{a}(\mathrm{t})$ & $\mathrm{b}(\mathrm{t})$ & $\mathrm{c}(\mathrm{t})$ \\
\hline 0 & 0.1 & 1 & 1.5 \\
0.05 & 0.100125 & 1.005 & 1.525636 \\
0.1 & 0.101 & 1.02 & 1.552585 \\
0.15 & 0.103375 & 1.045 & 1.580917 \\
0.2 & 0.108 & 1.08 & 1.610701 \\
0.25 & 0.115625 & 1.125 & 1.642013 \\
0.3 & 0.127 & 1.18 & 1.674929 \\
0.35 & 0.142875 & 1.245 & 1.709534 \\
0.4 & 0.164 & 1.32 & 1.745912 \\
0.45 & 0.191125 & 1.405 & 1.784156 \\
0.5 & 0.225 & 1.5 & 1.824361 \\
0.55 & 0.266375 & 1.605 & 1.866627 \\
0.6 & 0.316 & 1.72 & 1.911059 \\
0.65 & 0.374625 & 1.845 & 1.95777 \\
0.7 & 0.443 & 1.98 & 2.006876 \\
0.75 & 0.521875 & 2.125 & 2.0585 \\
0.8 & 0.612 & 2.28 & 2.11277 \\
0.85 & 0.714125 & 2.445 & 2.169823 \\
0.9 & 0.829 & 2.62 & 2.229802 \\
0.95 & 0.957375 & 2.805 & 2.292855 \\
1 & 1.1 & 3 & 2.359141 \\
\hline
\end{tabular}

\begin{tabular}{|c|r|r|c|}
\hline $\mathrm{t}$ & \multicolumn{1}{|c|}{$\mathrm{w} 1$} & \multicolumn{1}{c|}{$\mathrm{w} 2$} & $\mathrm{w} 3$ \\
\hline 0 & 0.12202 & 0.648329 & 0.229651 \\
0.05 & 0.122032 & 0.650441 & 0.227527 \\
0.1 & 0.122939 & 0.65207 & 0.224991 \\
0.15 & 0.125189 & 0.652457 & 0.222354 \\
0.2 & 0.129205 & 0.650917 & 0.219878 \\
0.25 & 0.135356 & 0.646922 & 0.217721 \\
0.3 & 0.143922 & 0.640169 & 0.215909 \\
0.35 & 0.155057 & 0.630604 & 0.214339 \\
0.4 & 0.168789 & 0.618394 & 0.212817 \\
0.45 & 0.185024 & 0.603858 & 0.211119 \\
0.5 & 0.203569 & 0.587395 & 0.209036 \\
0.55 & 0.224166 & 0.569422 & 0.206412 \\
0.6 & 0.246511 & 0.550334 & 0.203156 \\
0.65 & 0.270281 & 0.530484 & 0.199235 \\
0.7 & 0.29515 & 0.510183 & 0.194667 \\
0.75 & 0.320802 & 0.489692 & 0.189507 \\
0.8 & 0.346935 & 0.469231 & 0.183833 \\
0.85 & 0.373278 & 0.448984 & 0.177738 \\
0.9 & 0.399583 & 0.4291 & 0.171317 \\
0.95 & 0.425637 & 0.4097 & 0.164664 \\
1 & 0.451256 & 0.390876 & 0.157868 \\
\hline
\end{tabular}

The following expressions are best least-squares fits in some technical sense of each set of numerical data for the components shown in Table 1 and plotted in Figure 2 


$$
\begin{aligned}
& w_{1}(t) \approx \frac{1}{\sqrt{2 \pi}} \int_{-\infty}^{-0.053+1.15 \ln t} e^{-\frac{z^{2}}{2}} d z, t>0.4 \\
& w_{2}(t) \approx \frac{1}{\sqrt{2 \pi}} \int_{-\infty}^{-0.344-0.905 \ln t} e^{-\frac{z^{2}}{2}} d z, t>0.4 \\
& w_{3}(t) \approx e^{-0.956-0.979 t}, t>0.4
\end{aligned}
$$

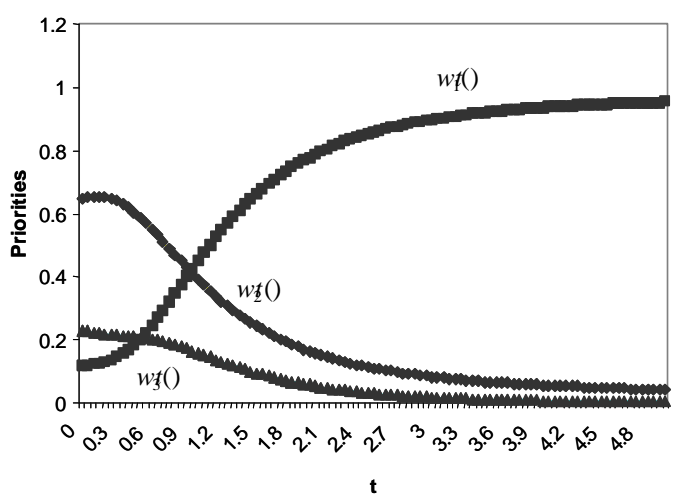

Figure 2 A Plot of the Numerical Estimate of the Components of the Principal Right Eigenvector

\section{Functional Dynamics: Analytic Solution of the Principal Eigenvalue Problem by Solving Algebraic Equations of Degree $n$}

Because we need to solve for the principal eigenvector, Perron theory gives us a way to obtain it more simply than by solving a high order algebraic equation or even by raising the matrix to arbitrarily large powers. On $\mathrm{p}$. 20 of Horn and Johnson's "Matrix Analysis" there is a brief discussion of the classical adjoint. Let $M=A-$ $\lambda_{\text {max }}(T) I$ be an $n$ by $n$ matrix, where $A$ is the pairwise comparisons matrix and $I$ is the identity matrix. What we now show is only useful when we know $\lambda_{\max }(T)$. Let $M^{\prime}$ be the matrix whose entry in the $i^{\text {th }}$ row and $j^{\text {th }}$ column is $(-1)^{i+j}$ times the $(n-1$ by $n-1)$ determinant of the matrix obtained by removing the $i^{\text {th }}$ column and $j^{\text {th }}$ row of $M$. Note the row/column transposition. Then $M M^{\prime}=\operatorname{det}(M) I$. Now $M$ has rank $n-1$. We note that in our situation, because its characteristic polynomial is of degree $n$ and vanishes at $\lambda_{\max }(T)$, the rank of $M$ cannot be more than $n$-1. By Perron, $\lambda_{\max }(T)$ is unique and cannot again be the root of a determinant of an $n$ 1 minor of $M$ and the rank of $M$ is precisely $n-1$. Thus $M M^{\prime}=0$ and every column of $M^{\prime}$ is an eigenvector of $M$ (belongs to the kernel of $M$ ). Since the kernel of $M$ is one-dimensional, all of the columns of $M$ ' must be proportional. They are also nonzero vectors because the rank of $M$ is $n-1$ that is "some" $n$-1byn-1 minor is nonsingular. This gives us another way of getting the principal right eigenvector of $A$ when we know $\lambda_{\max }(T)$. But for the supermatrix we already know that $\lambda_{\max }(T)=1$ which follows from:

$$
\begin{aligned}
& \max \sum_{j=1}^{n} a_{i j} \geq \sum_{j=1}^{n} a_{i j} \frac{w_{j}}{w_{i}}=\lambda_{\text {max }} \text { for } \max w_{i} \\
& \min \sum_{j=1}^{n} a_{i j} \leq \sum_{j=1}^{n} a_{i j} \frac{w_{j}}{w_{i}}=\lambda_{\text {max }} \text { for } \min w_{i}
\end{aligned}
$$

Thus for a row stochastic matrix we have $1=\min \sum_{j=1}^{n} a_{i j} \leq \lambda_{\max } \leq \max \sum_{j=1}^{n} a_{i j}=1$, thus $\lambda_{\max }=1$. 
The same type of argument applies when a matrix is column stochastic.

Here is an example of the foregoing ideas. The matrix $A$, its principal right eigenvector and its eigenvalues are:

$$
\left[\begin{array}{ccccc}
1 & 2 & 3 & 4 & 5 \\
\frac{1}{2} & 1 & 2 & 3 & 4 \\
\frac{1}{3} & \frac{1}{2} & 1 & 2 & 3 \\
\frac{1}{4} & \frac{1}{3} & \frac{1}{2} & 1 & 2 \\
\frac{1}{5} & \frac{1}{4} & \frac{1}{3} & \frac{1}{2} & 1
\end{array}\right]\left(\begin{array}{c}
0.418539 \\
0.262518 \\
0.159923 \\
0.097253 \\
0.061767
\end{array}\right)\left\{\begin{array}{c}
5.06808 \\
0.00498879+0.582755 \mathrm{i} \\
0.00498879-0.582755 \mathrm{i} \\
-0.0390289+0.068286 \mathrm{i} \\
-0.0390289-0.068286 \mathrm{i}
\end{array}\right\}
$$

$\lambda_{\max }(A)=5.06808$

The matrix $M=A-\lambda_{\max }(A) I$ is given by:

$$
\left[\begin{array}{ccccc}
-4.06808 & 2 & 3 & 4 & 5 \\
\frac{1}{2} & -4.06808 & 2 & 3 & 4 \\
\frac{1}{3} & \frac{1}{2} & -4.06808 & 2 & 3 \\
\frac{1}{4} & \frac{1}{3} & \frac{1}{2} & -4.06808 & 2 \\
\frac{1}{5} & \frac{1}{4} & \frac{1}{3} & \frac{1}{2} & -4.06808
\end{array}\right]
$$

Its adjoint matrix $M^{\prime}$ is:

$\left[\begin{array}{ccccc}136.49 & 214.908 & 353.393 & 580.105 & 924.877 \\ 85.6099 & 134.796 & 221.657 & 363.855 & 580.105 \\ 52.1526 & 82.116 & 135.031 & 221.657 & 353.393 \\ 31.7155 & 49.9371 & 82.116 & 134.796 & 214.908 \\ 20.1428 & 31.7155 & 52.1526 & 85.6099 & 136.49\end{array}\right]$

Note that $\mathrm{MM}^{\prime}=0=$

$$
\left[\begin{array}{ccccc}
-3.62 \times 10^{-12} & 8.35 \times 10^{-14} & 4.35 \times 10^{-14} & 3.83 \times 10^{-13} & 1.63 \times 10^{-13} \\
1.96 \times 10^{-14} & -3.61 \times 10^{-12} & 2.97 \times 10^{-14} & 1.20 \times 10^{-14} & 1.98 \times 10^{-14} \\
2.10 \times 10^{-14} & -2.05 \times 10^{-14} & -3.69 \times 10^{-12} & -9.44 \times 10^{-14} & 1.09 \times 10^{-13} \\
-2.64 \times 10^{-14} & -2.58 \times 10^{-14} & 1.58 \times 10^{-14} & -3.69 \times 10^{-12} & 4.67 \times 10^{-14} \\
1.36 \times 10^{-14} & 2.35 \times 10^{-14} & 3.04 \times 10^{-14} & 2.93 \times 10^{-14} & -3.66 \times 10^{-12}
\end{array}\right]
$$

Any column of M' such as the first gives principal right eigenvector given above in normalized form.

A technical problem that arises in this approach is that because of the time dependence of the coefficients of the matrix, it is difficult to generate the eigenvector of priorities in symbolic form if the order of the matrix is more than four. The reason is that in these cases one must solve a polynomial equation to obtain the principal 
eigenvalue and derive the corresponding principal eigenvector and we have expressions given below for both of these for $n \leq 4$. But for $n>4$ the story is complicated and has a long history in our time.

In this paper we give the analytic solution to derive the eigenvector for up to a $4 \times 4$ matrices and also give the solution of the quintic equation for the eigenvalues in closed form. The reader interested in this problem can contact this author for more general information on the solution of the quintic, sextic and septic equations. A technical problem that arises in this approach is that because of the time dependence of the coefficients of the matrix, it is difficult to generate the eigenvector of priorities in symbolic form if the order of the matrix is more than four. The reason is that in these cases one must solve a polynomial equation to obtain the principal eigenvalue and derive the corresponding principal eigenvector and we have expressions given below for both of these for $n \leq 4$. But for $n>4$ the story is complicated and has a long history in our time. Mathematica is a good program for providing the eigenvalues and the eigenvectors of matrices of large order. It would also be useful if one were to use it for time dependent judgments by taking different times, entering these numerical values of time, solving an entire decision problem numerically, and repeating the process. In the end the several decisionoutcome values can be approximated by time dependent curves.

\section{Quadratic Case}

To obtain the eigenvalue and eigenvectors of a 2 by 2 matrix, we must solve the problem

$$
\left[\begin{array}{cc}
1 & a(t) \\
1 / a(t) & 1
\end{array}\right]\left[\begin{array}{l}
w_{1}(t) \\
w_{2}(t)
\end{array}\right]=\lambda_{\max }\left[\begin{array}{l}
w_{1}(t) \\
w_{2}(t)
\end{array}\right]
$$

for which we know because the matrix is consistent that $\lambda_{\max }(t)=2$. For the eigenvector we need to solve the system of equations:

$$
\begin{aligned}
& w_{1}(t)+a(t) w_{2}(t)=2 w_{1}(t) \\
& w_{1}(t) / a(t)+w_{2}(t)=2 w_{2}(t)
\end{aligned}
$$

The first equation yields

$$
\begin{aligned}
& w_{1}(t)=a(t) w_{2}(t), \text { and because } w_{1}(t)+w_{2}(t)=1, \text { we have for our solution } \\
& w_{1}(t)=a(t) /[1+a(t)], w_{2}(t)=1 /[1+a(t)] .
\end{aligned}
$$

\section{Cubic Case}

$\lambda_{\max }=\left(a_{13} / a_{12} a_{23}\right)^{1 / 3}+\left(a_{12} a_{23} / a_{13}\right)^{1 / 3}+1$. If we define $\Delta=a_{12} a_{23}+a_{13}\left(\lambda_{\max }-1\right)$

and $D=a_{12} a_{23}+a_{13}\left(\lambda_{\max }-1\right)+\left(\lambda_{\max }-1\right) a_{23}+\left(a_{13} / a_{12}\right)-1+\left(1-\lambda_{\max }\right)^{2}$, we have

$$
\begin{aligned}
& w_{1}=\frac{\Delta}{D} \\
& w_{2}=\frac{\left(\lambda_{\max }-1\right) a_{23}+\left(a_{13} / a_{12}\right)}{D} \\
& w_{3}=\frac{-1+\left(1-\lambda_{\max }\right)^{2}}{D}
\end{aligned}
$$

\section{Quartic Case}

If we define 


$$
\begin{aligned}
& B=\left(\frac{a_{23} a_{34}}{a_{24}}+\frac{a_{24}}{a_{23} a_{34}}\right)+\left(\frac{a_{12} a_{24}}{a_{14}}+\frac{a_{14}}{a_{12} a_{24}}\right)+\left(\frac{a_{12} a_{23}}{a_{13}}+\frac{a_{13}}{a_{12} a_{23}}\right)+\left(\frac{a_{13} a_{34}}{a_{14}}+\frac{a_{14}}{a_{13} a_{34}}\right) \\
& C=3-\left(\frac{a_{12} a_{23} a_{34}}{a_{14}}+\frac{a_{14}}{a_{12} a_{23} a_{34}}\right)-\left(\frac{a_{12} a_{24}}{a_{13} a_{34}}+\frac{a_{13} a_{34}}{a_{12} a_{24}}\right)-\left(\frac{a_{14} a_{23}}{a_{13} a_{24}}+\frac{a_{13} a_{24}}{a_{14} a_{23}}\right)
\end{aligned}
$$

Then

and

$$
\begin{gathered}
\lambda_{\max }=\left[-8+\frac{B^{2}}{2}+8 C+\sqrt{\left[-\frac{4}{3}(C+3)\right]^{3}+\left(8-\frac{B^{2}}{2}-8 C\right)^{2}}\right]^{1 / 3} \\
+\left[-8+\frac{B^{2}}{2}+8 C-\sqrt{\left[-\frac{4}{3}(C+3)\right]^{3}+\left(8-\frac{B^{2}}{2}-8 C\right)^{2}}\right]^{1 / 3}
\end{gathered}
$$

where

$$
w_{1}=\frac{\overline{w_{1}}}{Q}, w_{2}=\frac{\overline{w_{2}}}{Q}, w_{3}=\frac{\overline{w_{3}}}{Q}, w_{4}=\frac{\overline{w_{4}}}{Q}
$$

$$
\begin{gathered}
Q=\left(\lambda_{\text {max }}-1\right)^{3}+\left(a_{14}+a_{34}+a_{24}\right)(\lambda-1)^{2}+\left[\left(a_{12} a_{24}-3\right)+\left(a_{13}+a_{23}\right) a_{34}+\left(\frac{1}{a_{12}}+\frac{1}{a_{13}}\right) a_{14}+\frac{a_{24}}{a_{23}}\right]\left(\lambda_{\text {max }}-1\right) \\
+\left[\left(a_{12} a_{23} a_{34}-a_{12}-a_{14}-a_{24}-a_{34}\right)+\left(\frac{a_{13} a_{24}}{a_{23}}+\frac{a_{13} a_{34}}{a_{12}}\right)+\frac{a_{14} a_{32}+a_{12} a_{24}}{a_{13}}+\frac{a_{14}-a_{13}}{a_{12} a_{23}}\right] \\
\overline{w_{1}}=a_{14}\left(\lambda_{\text {max }}-1\right)^{2}+\left(a_{12} a_{24}+a_{13} a_{34}\right)\left(\lambda_{\text {max }}-1\right)+\left(a_{12} a_{23} a_{34}+\frac{a_{13} a_{24}}{a_{23}}-a_{14}\right) \\
\overline{w_{2}}=a_{24}\left(\lambda_{\max }-1\right)^{2}+\left(a_{23} a_{34}+\frac{a_{14}}{a_{12}}\right)\left(\lambda_{\text {max }}-1\right)+\left(\frac{a_{13} a_{34}}{a_{12}}+\frac{a_{14} a_{23}}{a_{13}}-a_{24}\right) \\
\overline{w_{3}}=a_{34}\left(\lambda_{\max }-1\right)^{2}+\left(\frac{a_{24}}{a_{23}}+\frac{a_{14}}{a_{13}}\right)\left(\lambda_{\max }-1\right)+\left(\frac{a_{14}}{a_{12} a_{23}}+\frac{a_{12} a_{24}}{a_{13}}-a_{34}\right) \\
\overline{w_{4}}=\left(\lambda_{\max }-1\right)^{3}-3\left(\lambda_{\text {max }}-1\right)-\left(\frac{a_{12} a_{23}}{a_{13}}+\frac{a_{13}}{a_{12} a_{23}}\right)
\end{gathered}
$$

REMARK It is easy to see from this solution that if any coefficient is increased (decreased) in a given row of the pairwise comparison matrix the value of the eigenvector component corresponding to that row is increased (decreased) relative to the remaining components. This property holds for a reciprocal matrix.

\section{The Quintic and Higher Order Cases}

Algebraic Equations by Theta Constants, in Tata Lectures on Theta II, D. Mumford, ed., pp. 3.261-3.272, Birkhaüser, Boston, 1984, do not require the use of Tschirnhausen transformations. Let $f(x)=a_{0} x^{n}+a_{1} x^{n-1}+\ldots+a_{n}=0, a_{0} \neq 0$ be an algebraic equation irreducible over a subfield of the complex numbers. A root of this equation can be expressed in terms of theta functions of zero argument involving the period matrix derived from one of two types of hyper-elliptic integrals. 
Bernd Sturmfels gives the solution of the quintic equation in term using the finest of the $2^{n-1}$ triangulations of the hypergeometric differential equations and corresponding $2^{n-1}$ series solutions. The roots of the quintic $a_{5} x^{5}+a_{4} x^{4}+a_{3} x^{3}+a_{2} x^{2}+a_{1} x+a_{0}=0$ one of which is the principal eigenvalue that may also be obtained in other ways are given by an infinite series and the question about such expansions is whether the series always converge. We have:

$$
\begin{aligned}
& X_{1,-1}=-\left[\frac{a_{0}}{a_{1}}\right], X_{2,-1}=-\left[\frac{a_{1}}{a_{2}}\right]+\left[\frac{a_{0}}{a_{1}}\right], X_{3,-1}=-\left[\frac{a_{2}}{a_{3}}\right]+\left[\frac{a_{1}}{a_{2}}\right], \\
& X_{4,-1}=-\left[\frac{a_{3}}{a_{4}}\right]+\left[\frac{a_{2}}{a_{3}}\right], X_{5,-1}=-\left[\frac{a_{4}}{a_{5}}\right]+\left[\frac{a_{3}}{a_{4}}\right] .
\end{aligned}
$$

Each bracket represents a power series with a monomial in the bracket as its first term:

$$
\begin{aligned}
& {\left[\frac{a_{0}}{a_{1}}\right]=\frac{a_{0}}{a_{1}}+\frac{a_{0}^{2} a_{2}}{a_{1}^{3}}-\frac{a_{0}^{3} a_{3}}{a_{1}^{4}}+2 \frac{a_{0}^{3} a_{2}^{2}}{a_{1}^{5}}+\frac{a_{0}^{4} a_{4}}{a_{1}^{5}}-5 \frac{a_{0}^{4} a_{2} a_{3}}{a_{1}^{6}}-\frac{a_{0}^{5} a_{5}}{a_{1}^{6}}+\ldots} \\
& {\left[\frac{a_{1}}{a_{2}}\right]=\frac{a_{1}}{a_{2}}+\frac{a_{1}^{2} a_{3}}{a_{2}^{3}}-\frac{a_{1}^{3} a_{4}}{a_{2}^{4}}-3 \frac{a_{0} a_{1}^{2} a_{5}}{a_{2}^{4}}+2 \frac{a_{1}^{3} a_{3}^{3}}{a_{2}^{5}}+\frac{a_{1}^{4} a_{5}}{a_{2}^{5}}-5 \frac{a_{1}^{4} a_{3} a_{4}}{a_{2}^{6}}+\ldots} \\
& {\left[\frac{a_{2}}{a_{3}}\right]=\frac{a_{2}}{a_{3}}-\frac{a_{0} a_{5}}{a_{3}^{2}}-\frac{a_{1} a_{4}}{a_{3}^{2}}+2 \frac{a_{1} a_{2} a_{5}}{a_{3}^{3}}+\frac{a_{2}^{2} a_{4}}{a_{3}^{3}}-\frac{a_{2}^{3} a_{5}}{a_{3}^{4}}+2 \frac{a_{2}^{3} a_{4}^{2}}{a_{3}^{5}}+\ldots} \\
& {\left[\frac{a_{3}}{a_{4}}\right]=\frac{a_{3}}{a_{4}}-\frac{a_{2} a_{5}}{a_{4}^{2}}+\frac{a_{3}^{2} a_{5}}{a_{4}^{3}}+\frac{a_{1} a_{5}^{2}}{a_{4}^{3}}-3 \frac{a_{2} a_{3} a_{5}^{2}}{a_{4}^{4}}-\frac{a_{0} a_{5}^{3}}{a_{4}^{4}}+4 \frac{a_{1} a_{3} a_{5}^{3}}{a_{4}^{5}}+\ldots} \\
& {\left[\frac{a_{4}}{a_{5}}\right]=\frac{a_{4}}{a_{5}} .}
\end{aligned}
$$

\section{Value of Pairwise Comparisons - an Example}

The following example shows that by making pairwise comparisons we learn much more about the detail of a dynamic priority function than if we were to simply guess at each component of the eigenvector components individually.

\section{Family Spending Time at Home}

Let us consider the simple case of a family with a father, a mother, and a child. Obviously the amount of time the child spends at home will depend on his age. An infant will spend the same amount of time at home as the mother and then, as he grows older, he will spend progressively less time at home compared to the mother. We assume that the mother does not go out to work.

If we were to compare the length of time spent at home by mother and child and plot this relation as a function of time (i.e., as the child grows older), we would get the type of curve shown in Figure 3. 


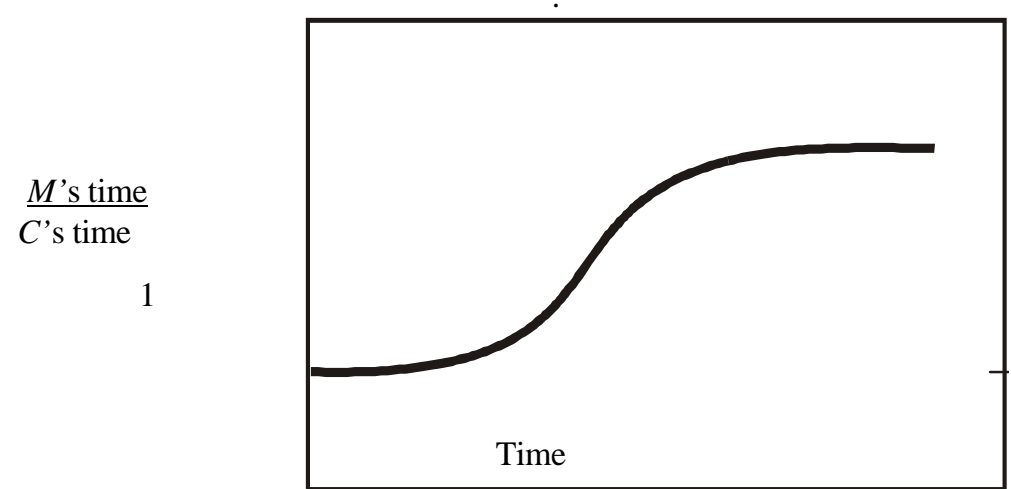

Figure 3 Relative Time at Home - Mother to Child

Thus the curve begins with the home maker mother and child spending the same amount of time, then the ratio of mother's to child's time increases until it levels off by the time the child is in her/his mid-teens. Comparison of father to child times yields a relationship that is a mirror image of the above -reflected about a horizontal axis halfway up the curve. This is illustrated in Figure 4. The relative length of time spent by father and mother would not vary too much and could be expected to be fairly constant.

If we were to make a pairwise comparison of the different lengths of time spent at home by the different members of the family, we would get a sequence of comparison matrices each corresponding to a particular period of time.

Consider the time period corresponding to the child's age 0-4 years. If we were to exclude, say, eight hours of the night, we would expect the mother and child to spend about two to three times the length of time the father spends at home. The mother and child would of course spend the same amount of time.

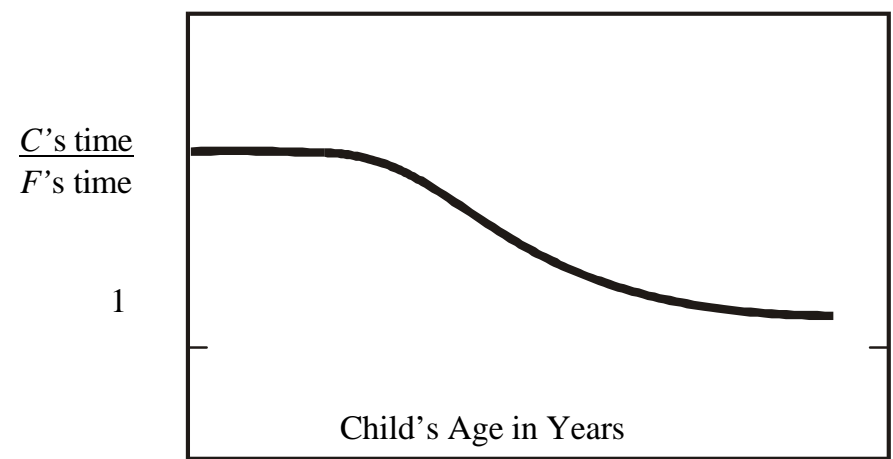

Figure 4 Relative Time at Home - Child to Father

This would give rise to the following matrix:

$$
\begin{aligned}
& \begin{array}{c}
F \\
C
\end{array} \quad\left[\begin{array}{lcc}
1 & M & C \\
2.5 & 1 / 2.5 & 1 / 2.5 \\
2.5 & 1 & 1 \\
& 1 & 1
\end{array}\right] \\
& \lambda_{\max }=3.0, \quad \text { C.I. }=0.0, \quad \text { C.R. }=0.0
\end{aligned}
$$

This yields the following eigenvector for their relative times at home: 
$F: 0.167$

M: 0.417

$C: 0.417$

That would seem to be a reasonable estimate of the proportions of time each spends at home. Around the age of four the child begins school, so there is a sudden change in the relative proportions of time spent at home by mother and child and by father and child.

Moving to the time dependent situation, we can express the varying proportions in a single matrix as shown below:

$$
\begin{gathered}
F \\
C
\end{gathered}\left[\begin{array}{ccc}
F & M & C \\
1 & 1 / 2 & 1 /(3-\ln t / 2) \\
2 & 1 & 0.4+\ln t / 2 \\
3-\ln t / 2 & 1 /(0.4+\ln t / 2) & 1
\end{array}\right]
$$

The variable $t$ denotes the child's age ranging between 4 and 16 years. This matrix, along with the previous one, gives rise to the curves in Figure 5, Figure 6 and Figure 7 that depict the corresponding pairwise comparisons as time varies from zero to 16 years.

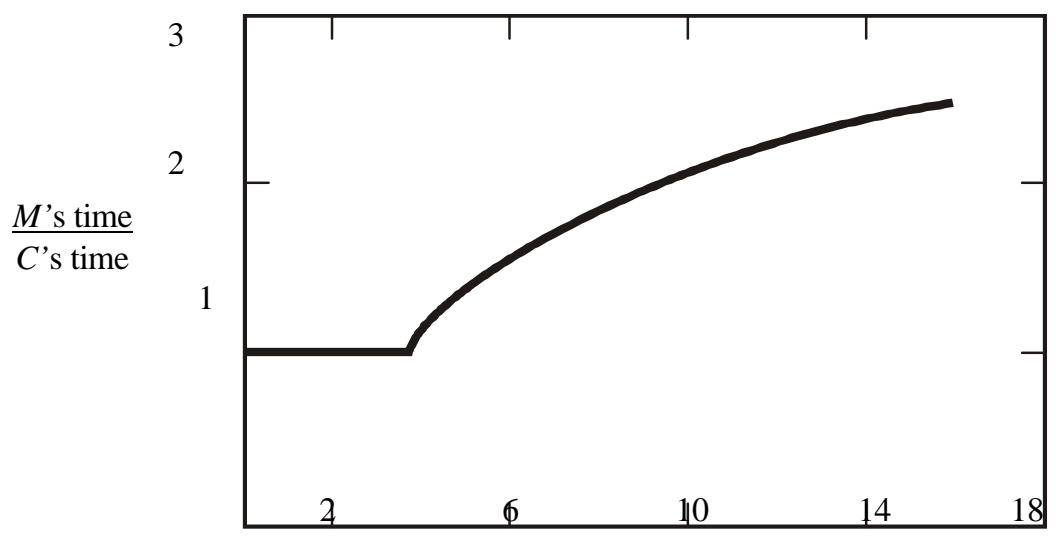

Figure 5 Mother to Child: age 0-16 years

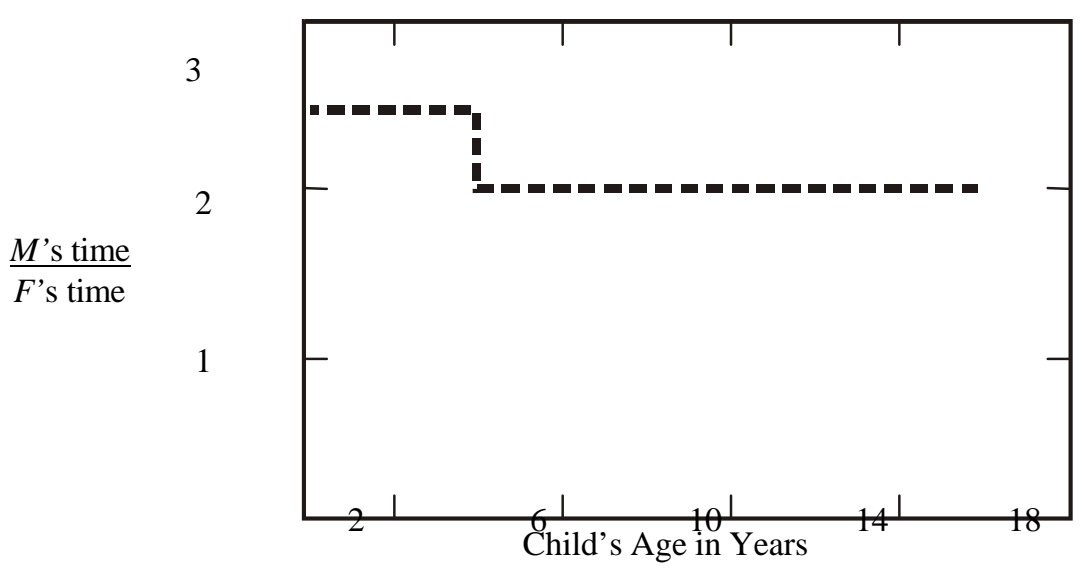

Figure 6 Mother to Father: Age 0-16 years 
The solution of the maximum eigenvalue problem corresponding to these pairwise comparison curves for ( $4 \leq t$ $\leq 16)$ is

$$
\lambda_{\max }=1+\left[\frac{2}{(3-\ln t / 2)(0.4+\ln t / 2)}\right]^{1 / 3}+\left[\frac{(3-\ln t / 2)(0.4+\ln t / 2)}{2}\right]^{1 / 3}
$$

The corresponding eigenvector is given by

$$
w_{1}=\Delta / D ; w_{2}=\left[\left(\lambda_{\max }-1\right)(0.4+\ln t / 2)+\frac{2}{3-\ln t / 2}\right] / D ; w_{3}=\left[-1+(-2)^{2}\right] / D
$$

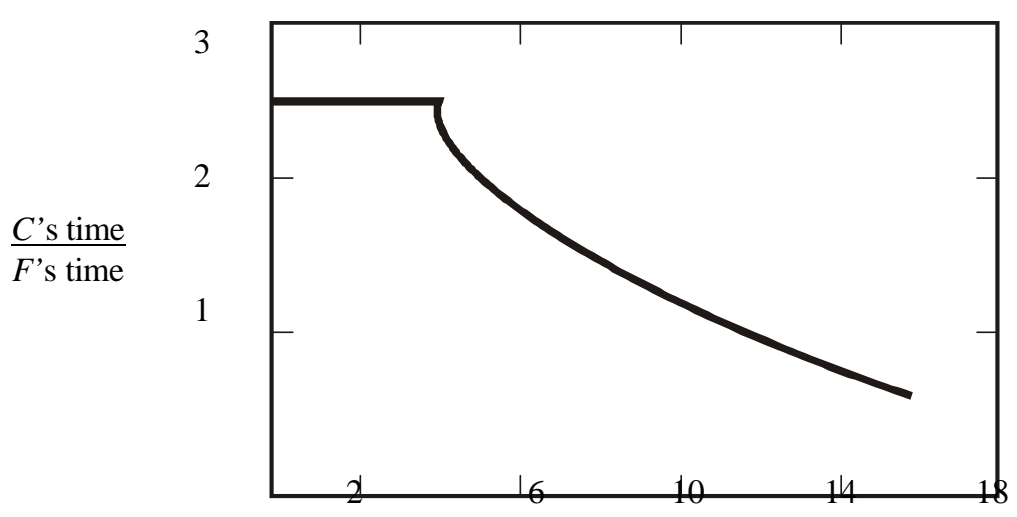

Child's Age in Years

Figure 7 Child to Father: age 0-16 years

where

$$
\begin{gathered}
\Delta=0.5(0.4+\ln t / 2)+\frac{\lambda_{\text {max }}-1}{3-\ln t / 2} \\
D=\left(\lambda_{\text {max }}-0.5\right)(0.4+\ln t / 2)+\frac{\lambda_{\text {max }}+1}{3-\ln t / 2}-1+\left(1-\lambda_{\text {max }}\right)^{2}
\end{gathered}
$$

As the child finishes school, he begins spending even less time at home than the father. The proportions once again become fairly constant and are reflected in the following (consistent) pairwise comparison matrix:

$$
\begin{gathered}
F \\
M \\
C
\end{gathered}\left[\begin{array}{ccc}
F & M & C \\
1 & 0.5 & 1.25 \\
2 & 1 & 2.5 \\
0.8 & 0.4 & 1
\end{array}\right]
$$

The eigenvector solution is given by:
$F: 0.263$
M: 0.526
C: 0.211 
Plotting these results together for $0 \leq t \leq 4,4 \leq t \leq 16$, and $16 \leq t$ gives a realistic representation of the relative time, with respect to all others, which each spends at home (seeFigure 8).

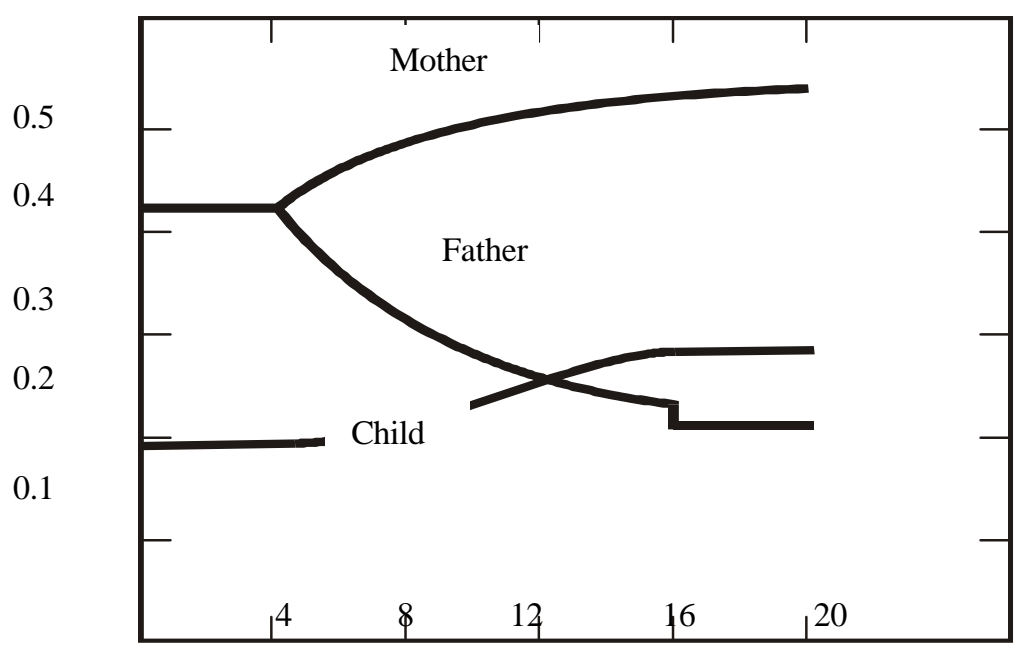

Child's Age in Years

Figure 8 Relative Proportion of Time Spent at Home

\section{Pairwise Comparison Judgments and Scale - General Discussion}

Recall that in making paired comparisons of intangibles, we use the smaller or lesser element as a unity and estimate the larger one as a multiple of it. We can also do that in dynamic judgments by simply introducing a function to estimate how many times more is the larger element than the unit. In this case there may be a change of unit of the comparison if the larger element becomes the smaller and must serve as the unit. Just as we used absolute numbers to enter into the comparison matrix in the static case, we need to enter basic functions to represent judgments in the dynamic case. What typical functions can we use that would for example approximate well the functions of the family example above? I do not have a conclusive answer to that question, if indeed there is an answer to it. For the pairwise comparison judgments one may attempt to fit one of the functions given in Table 2 to the dynamic judgments. These functions have been left in parametric form so that the parameter may be set for the particular comparison, hopefully meeting the homogeneity requirement of the1-9 scale used in the discrete case as a limit on the range of values. They, and combinations of them, reflect our intuitive feeling about relative change in trend: constant, linear, polynomial, logarithmic, exponential, oscillatory, and finally discrete that allows for sudden change like a Dirac delta function.

The following is an example given by Andreichicov and Andreichicova, 1999. They assumed that the preferences for alternatives at the bottom with respect to the criteria in the fourth hierarchy level of Figure 9 would remain constant in the future. However, they assumed that preferences for the factors located at the second hierarchy level would vary.

The relative importance of the criteria for each actor also would be subject to modification in the future. The filling of pairwise comparison matrices as the dynamic task was made as follows. There were $(n-1)$ cells in a matrix selected, where the functions describing changes of appropriate preferences were made. The preferences at the time coincided with preferences in the static task. At the following instants the values for the other $\left(n^{2}-\right.$ $2 n+1)$ preferences were calculated on the basis of the $(n-1)$ functions given (Auto). Thus there was no problem of inconsistency during solution of the dynamic problem. Forming the functions was produced experimentally with the help of the software developed by the authors. 
Table 2 This Author's Idea of A Mathematician's Formulation of a Dynamic Judgment Scale

\begin{tabular}{|c|c|c|}
\hline $\begin{array}{l}\text { Time-dependent } \\
\text { importance intensity }\end{array}$ & Description & Explanation \\
\hline$a$ & Constant for all $t, 1 \leq a \leq 9$ an integer & No change in relative standing \\
\hline$a_{1} t+a_{2}$ & $\begin{array}{l}\text { Linear relation in } t \text {, increasing or decreasing to a point } \\
\text { and then a constant value thereafter. Note that the } \\
\text { reciprocal is a hyperbola. }\end{array}$ & $\begin{array}{l}\text { Steady increase in value of one } \\
\text { activity over another }\end{array}$ \\
\hline$b_{1} \log (t+1)+b_{2}$ & $\begin{array}{l}\text { Logarithmic growth up to a certain point and constant } \\
\text { thereafter }\end{array}$ & $\begin{array}{l}\text { Rapid increase (decrease) } \\
\text { followed by slow increase } \\
\text { (decrease) }\end{array}$ \\
\hline$c_{1} e^{c_{2} t}+c_{3}$ & $\begin{array}{l}\text { Exponential growth (or decay if } c_{2} \text { is negative) to a } \\
\text { certain point and constant thereafter (not reciprocal of } \\
\text { case } c_{2} \text { is negative is the logistic } S \text {-curve) }\end{array}$ & $\begin{array}{l}\text { Slow increase (decrease) } \\
\text { followed by rapid increase } \\
\text { (decrease) }\end{array}$ \\
\hline$d_{1} t^{2}+d_{2} t+d_{3}$ & $\begin{array}{l}\text { A parabola giving a maximum or minimum (depending } \\
\text { on } d_{1} \text { being negative or positive) with a constant value } \\
\text { thereafter. (May be modified for skewness to the right or } \\
\text { left) }\end{array}$ & $\begin{array}{l}\text { Increase (decrease) to maximum } \\
\text { (minimum) and then decrease } \\
\text { (increase) }\end{array}$ \\
\hline$e_{1} t^{n} \sin \left(t+e_{2}\right)+e_{3}$ & Oscillatory & $\begin{array}{l}\text { Oscillates depending on } \mathrm{n}, \mathrm{n}>0 \\
(n \leq 0) \text { with decreasing } \\
\text { (increasing) amplitude }\end{array}$ \\
\hline Catastrophes & Discontinuities indicated & Sudden changes in intensity \\
\hline
\end{tabular}

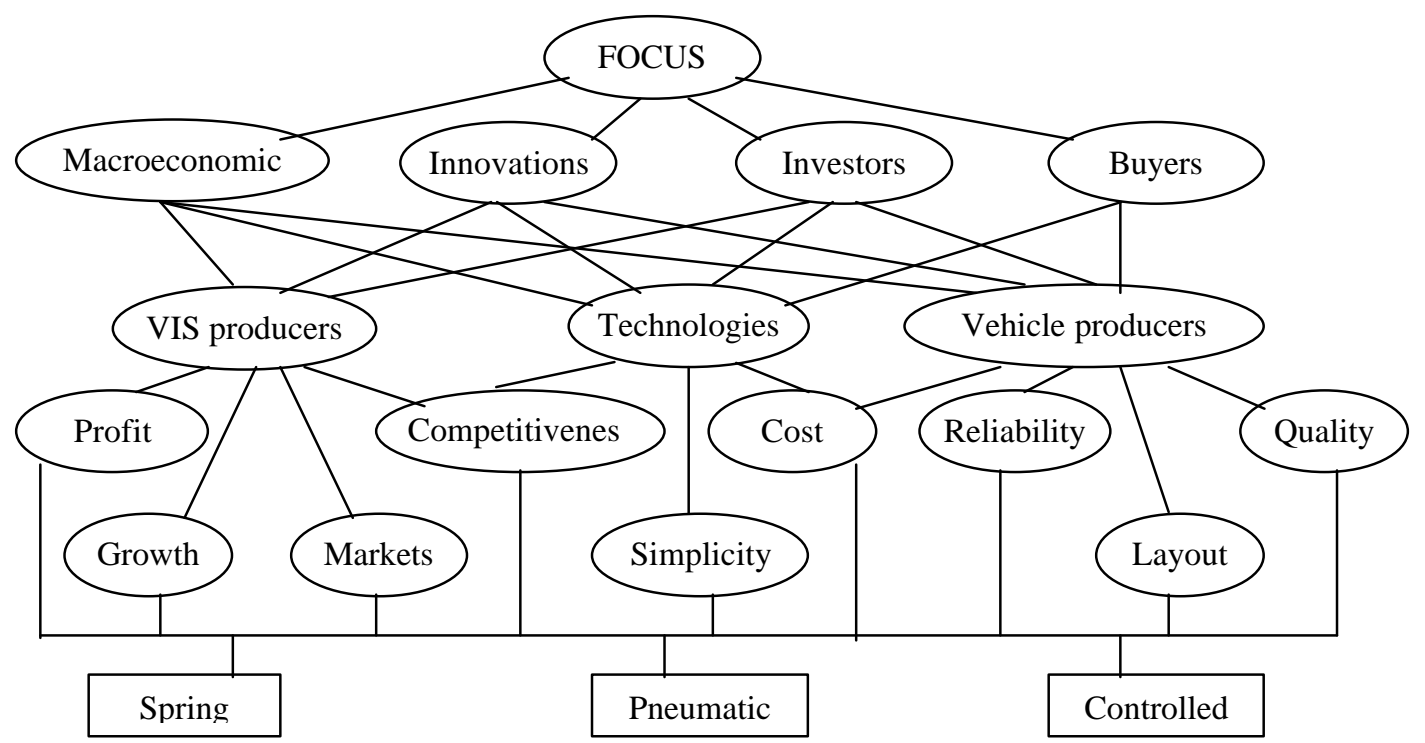

Figure 9 Hierarchy for Dynamic Evaluation 
Table 3 Priority Vectors for Criteria in the Static Problem

\begin{tabular}{lllll}
\hline \hline & Macroeconomic & Innovations & Investments & Buyers \\
FOCUS & 0.380 & 0.085 & 0.466 & \\
\hline & VIS producers & Vehicles producers & Technologies & \\
Macroeconomic & 0.109 & 0.582 & 0.309 & \\
Innovations & 0.105 & 0.258 & 0.637 & \\
Investments & 0.167 & 0.740 & 0.094 & \\
Buyers & 0.167 & 0.833 & & Markets \\
& Profit & Growth & Competitiveness & 0.186 \\
VIS producers & 0.532 & 0.097 & 0.186 & Layout \\
\hline & Quality & Cost & Reliability & 0.402 \\
\hline Vehicles producers & 0.143 & 0.402 & 0.054 & \\
Technologies & Competitiveness & Cost & Simplicity & \\
\hline \hline
\end{tabular}

The dynamic paired comparison matrices were the following:

\begin{tabular}{|c|c|c|c|c|c|c|c|}
\hline FOCUS & Macroeconomic & \multicolumn{2}{|c|}{ Innovations } & \multicolumn{2}{|c|}{ Investments } & \multicolumn{2}{|c|}{ Buyers } \\
\hline Macroeconomic & $\mid 1$ & \multicolumn{2}{|c|}{$1 / 0.25 e^{0.6 t}$} & \multicolumn{2}{|c|}{ Auto } & \multicolumn{2}{|c|}{$1 /\left(0.2-0.05 t+0.08 t^{2}\right)$} \\
\hline Innovations & $0.25 e^{0.6 t}$ & \multicolumn{2}{|c|}{1} & \multicolumn{2}{|c|}{$0.2-0.18 t+0.15 t^{2}$} & \multicolumn{2}{|c|}{ Auto } \\
\hline Investments & auto & \multicolumn{2}{|c|}{$1 /\left(0.2-0.18 t+0.15 t^{2}\right)$} & \multicolumn{2}{|c|}{1} & \multicolumn{2}{|l|}{ Auto } \\
\hline Buyers & $0.2-0.05 t+0.08 t^{2}$ & \multicolumn{2}{|c|}{ Auto } & \multicolumn{2}{|c|}{ Auto } & \multicolumn{2}{|l|}{1} \\
\hline VIS producers & Profit & \multicolumn{2}{|c|}{ Growth } & \multicolumn{3}{|c|}{ Competitiveness } & Markets \\
\hline Profit & $\mid 1$ & \multicolumn{2}{|c|}{$1 /\left(0.2+0.05 t+0.025 t^{2}\right)$} & \multicolumn{3}{|c|}{$1 / 0.333 e^{0.43 t}$} & $1 / 0.333 e^{0.43 t}$ \\
\hline Growth & $0.2+0.05 t+0.025 t^{2}$ & \multicolumn{2}{|c|}{1} & \multicolumn{3}{|c|}{ Auto } & Auto \\
\hline Competitiveness & $0.333 e^{0.43 t}$ & \multicolumn{2}{|l|}{ Auto } & \multicolumn{3}{|l|}{1} & Auto \\
\hline Markets & $0.333 e^{0.43 t}$ & \multicolumn{2}{|l|}{ Auto } & Auto & & 1 & \\
\hline Vehicles produce & Quality & & Cost & & Reliab & ility & Layout \\
\hline Quality & & & $0.333+0.4 t$ & $.04 t^{2}$ & $3-1.15$ & $t+0.18 t^{2}$ & $0.333+0.15 t$ \\
\hline Cost & $1 /(0.333+0.4 t$ & $\left.t+0.04 t^{2}\right)$ & 1 & & Auto & & Auto \\
\hline Reliability & $1 /(3-1.15 t+0$ & $\left.18 t^{2}\right)$ & Auto & & 1 & & Auto \\
\hline Layout & $1 /(0.333+0.15$ & & Auto & & Auto & & 1 \\
\hline
\end{tabular}

Table 4 Priority Vectors for Alternatives in the Static Problem

\begin{tabular}{cccc}
\hline \hline & Coil spring & Pneumatic & Controlled \\
\hline Profit & 0.648 & 0.230 & 0.122 \\
Growth & 0.143 & 0.429 & 0.429 \\
Competitiveness & 0.075 & 0.333 & 0.592 \\
Markets & 0.109 & 0.582 & 0.309 \\
Quality & 0.066 & 0.319 & 0.615 \\
Cost & 0.751 & 0.178 & 0.070 \\
Reliability & 0.637 & 0.105 & 0.258 \\
Layout & 0.105 & 0.258 & 0.637 \\
Simplicity & 0.751 & 0.178 & 0.070 \\
\hline \hline
\end{tabular}


The other paired comparison matrices were the same as in the static problem. The results are shown in Figure 10.

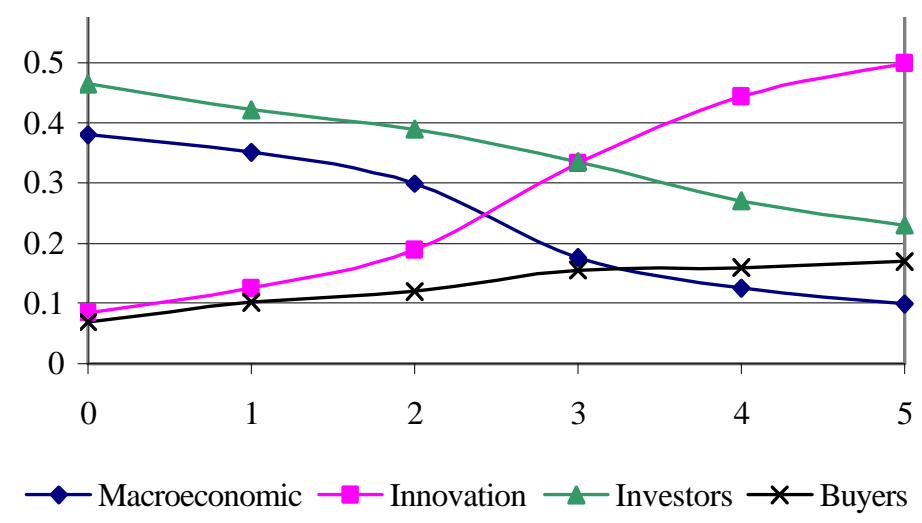

Figure 10 Change in Factor Priorities over Time

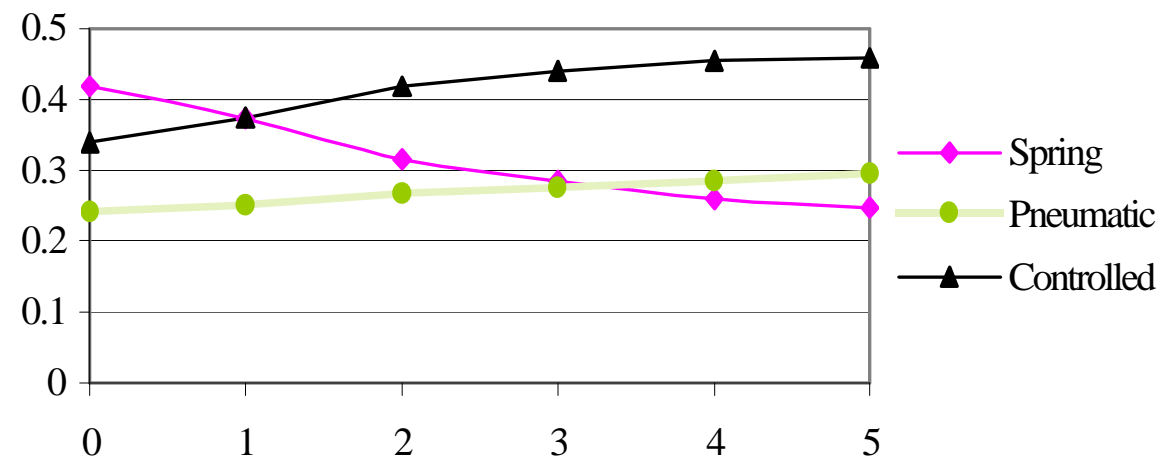

Figure 11 Change in Each Alternative's Priorities over Time

The priority of innovation in Russia is expected to increase in the future. The buyer's priority will also increase slightly. The investor's importance and macroeconomic influence will decrease. These modifications and the changes in the importance of the criteria for producers will result in a change in the order established for the alternatives. The spring coil, which is simple and cheap, moves from first place to last place. The best alternative in the future will be VIS controlled, which has high vibroisolation quality and high cost. The low priority of the pneumatic VIS in both cases might be explained by its relatively high cost and low reliability.

\section{Fundamental Scale of Relative Change- On The Rate of Change of Intangibles!}

Let us now turn to a more practical way of creating a fundamental scale that is in harmony with any person's way of looking ahead to make comparisons. As in the discrete case, because we have intangibles we have no functions to describe their behavior absolutely, but we can compare them to derive scales of relative change over time. We have no problem to deal with tangibles whose behavior over time is known though a function-oftime expression for each.

The question is how to generalize this idea to the dynamic case. Two kinds of data need to be generated. One is the initial point $x(0)$ of the curve of relative change. Here we ask the same question as in the discrete case: which of the two dominates the other with regard to a common attribute they share? The 
other question has to do with their perceived relative change: which of the two is perceived to change more than the other with respect to a common attribute they share? This second question is answered with perhaps different estimates for different time periods.

As in the discrete case, an individual has the ability to recognize distinctions among high, medium and low and for each of these also three distinctions of high, medium and low. In the case of static judgments that deal with numbers we had the nine values of the fundamental scale 1-9. We assume for the moment that when we compare two (tangible) activities each described by a curve as a function of time that we can make these nine distinctions in their rates of change relative to one another. How can each of these curves vary so that we would be able to divide the relative variation between them into recognizable nine categories over time? We note that in the simplest case where both functions are linear in time and their relative variation expressed by their ratio is a hyperbola over time. A hyperbola has a simple but rather sophisticated interpretation. What are different possible interpretations of variations (ratios) between two functions? To deal with such concepts effectively we need to think of time in two ways, the hard way as a tangible measured on a clock and make judgments over known perhaps uniform time intervals, or the soft way as an intangible divided according to our feelings about it such as short term, mid term and long term. This author's idea of a practical-minded person's representation of a dynamic judgment scale of relative changes is shown in Table 5.

Table 5 Formulation of a Dynamic Judgment Scale

\begin{tabular}{|l|}
\hline \multicolumn{2}{|c|}{ Comparison of A over $\mathrm{B}$} \\
\hline Extreme Increase \\
\hline Very Strong Increase: $\mathrm{x}(\mathrm{t})=2.414 \mathrm{t}$ \\
\hline Strong Increase: $\mathrm{x}(\mathrm{t})=\mathrm{t}$ \\
\hline Moderate Increase $; \mathrm{x}(\mathrm{t})=0.414 \mathrm{t}$ \\
\hline Equal; $\mathrm{x}(\mathrm{t})=1$ \\
\hline Moderate Decrease: $\mathrm{x}(\mathrm{t})=-0.414 \mathrm{t}$ \\
\hline Strong Decrease: $\mathrm{x}(\mathrm{t})=-\mathrm{t}$ \\
\hline Very Strong Decrease: $\mathrm{x}(\mathrm{t})=-2.414 \mathrm{t}$ \\
\hline Extreme Decrease \\
\hline
\end{tabular}

\begin{tabular}{|l|c|c|c|}
\hline \multicolumn{1}{|c|}{ Comparison of A over B } & $\begin{array}{c}\text { Slope of Line } \\
\left(0 \leq \theta \leq 90^{\circ}\right)\end{array}$ & $\begin{array}{c}\text { Slope of Line } \\
\left(0 \leq \theta \leq 45^{\circ}\right)\end{array}$ & $\begin{array}{c}\text { Slope of Line } \\
\left(0 \leq \theta \leq 22.5^{\circ}\right.\end{array}$ \\
\hline Extreme Increase & $\infty$ & 1 & 0.414 \\
\hline Very Strong Increase & 2.414 & 0.668 & 0.303 \\
\hline Strong Increase & 1 & 0.414 & 0.199 \\
\hline Moderate Increase & 0.414 & 0.199 & 0.098 \\
\hline Equal & 0 & 0 & 0 \\
\hline Moderate Decrease & -0.414 & -0.199 & -0.098 \\
\hline Strong Decrease & -1 & -0.414 & -0.199 \\
\hline Very Strong Decrease & -2.414 & -0.668 & -0.303 \\
\hline Extreme Decrease & $-\infty$ & -1 & -0.414 \\
\hline
\end{tabular}

An application of it over time is shown in Table 6. 
Table 6 Different Possible Formulations and Variations using the Basic Scale in Table 5

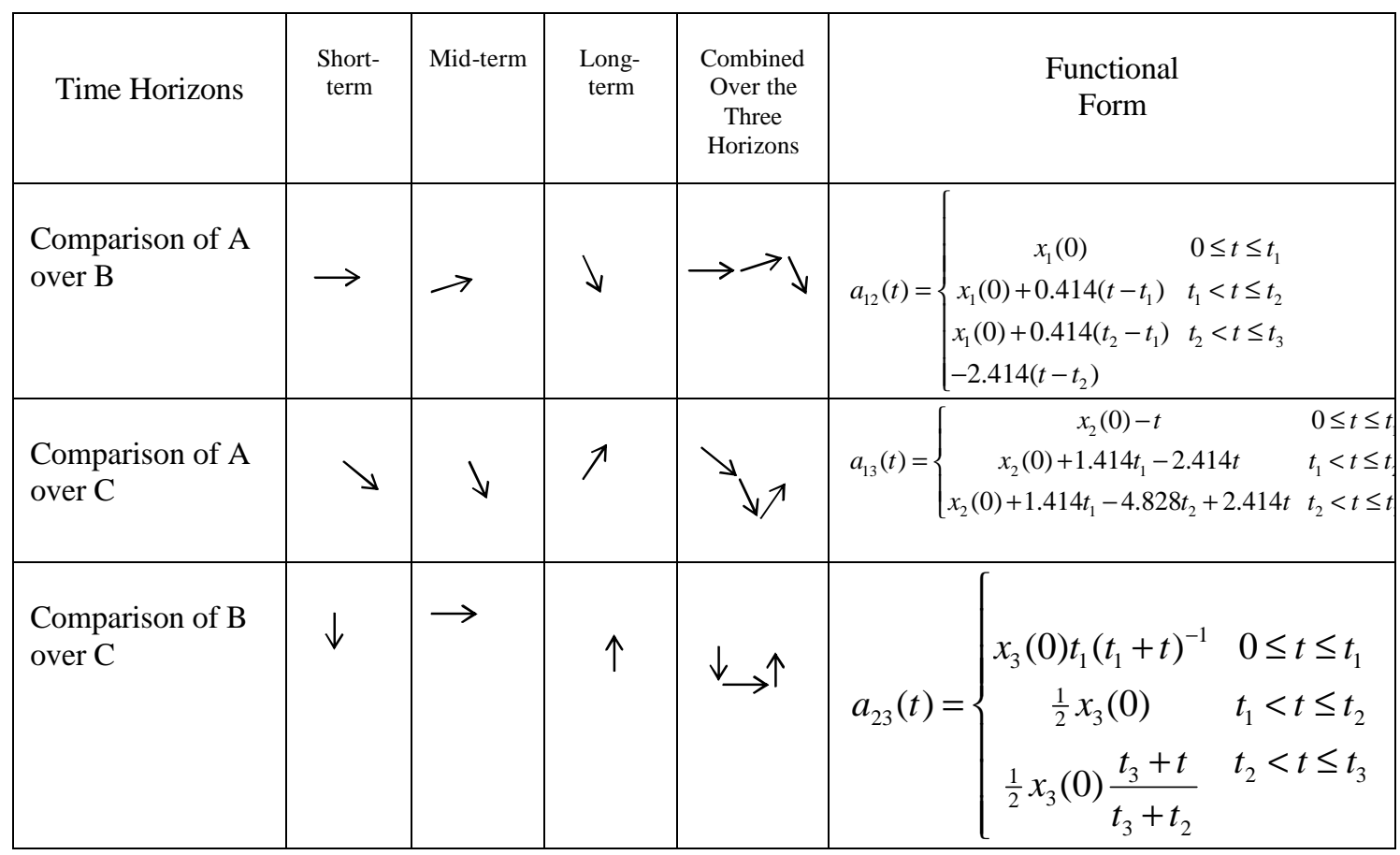

As in the 1-9 scale, time horizons may be divided into 9 periods starting with the present time and relative increases and decreases further refined. Here a function representing judgments may descend below $x(t)=$ 1 and thus the coefficient may include a description of both dominance and being dominated. Its transpose is the reciprocal function. Because we do not wish to use upward or downward arrows for an entire judgment over a time horizon interval, we can replace such a "Dirac" type function by a curve that rises or falls sharply and continuously over the interval. Examples are $(t-a)^{-1},|t-a|^{-1}$ and $(t-a)^{-2}$. Homogeneity needs to be maintained not only for magnitudes but also for the angle of relative change. The angle span for homogeneity may be less than 90 degrees. Perhaps it should be closer to a radian $360 / \pi=57.296^{\circ}$ and its division into ranges of just noticeable increases in slope or angle. There needs to be a theoretical way to justify the bound on the homogeneity of relative change. When exceeded, one may have to use clustering as in the comparison of different magnitudes.

Note that because the time horizons are not well specified over time, instead of connecting their end points to make a continuous curve, one may select the mid point of a time horizon to draw each line segment and take their points of intersection as the positions where the composite curve connects.

We now show by an example that real life situations reveal themselves along similar lines to the line segments we combined to put together decisions made over short, medium and long term time horizons. The example has to deal with the earnings and projected electricity use by the three companies involved (taken from the internet) as shown in Figure 12. 
IBM vs. Microsoft

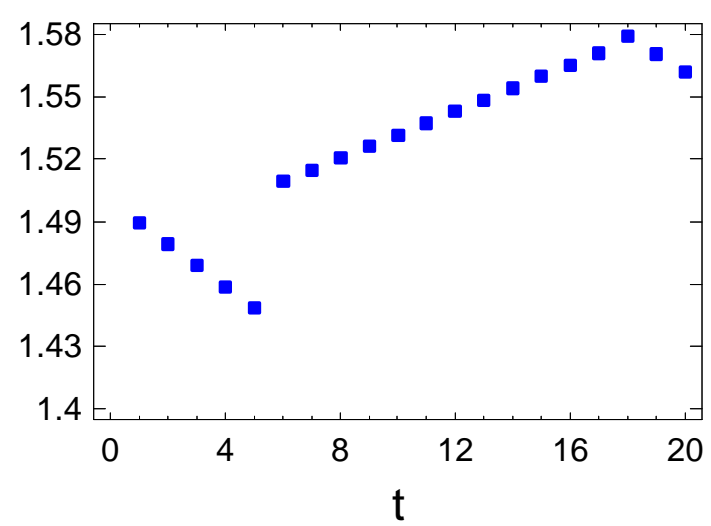

IBM vs. AT\&T

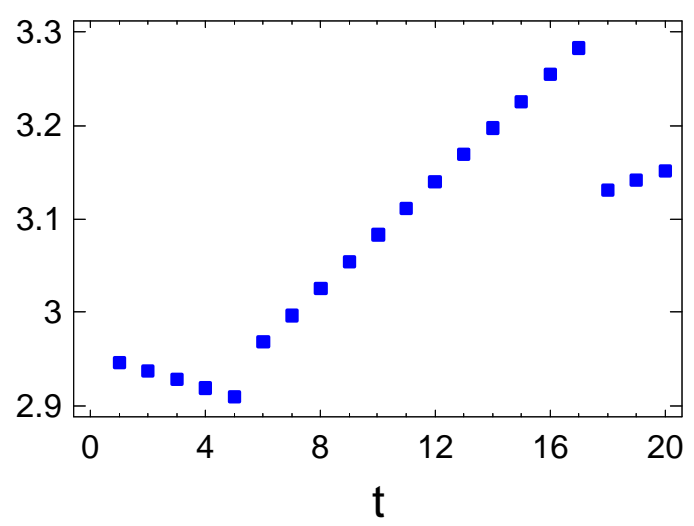

Microsoft vs. AT\&T

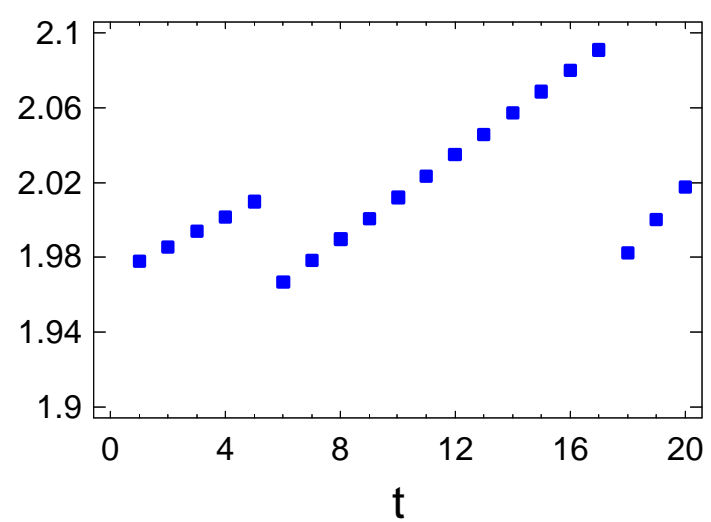

Figure 12 Relative Earnings of Software Companies 
Judgments fitted with linear approximations:

\begin{tabular}{|c|c|c|c|}
\hline Short Term & $I B M$ & $M S F T$ & $A T \& T$ \\
\hline$I B M$ & {$\left[\begin{array}{ll}1 & 1.45\end{array}\right.$} & $-0.0103 t$ & $2.955-0.0089 t$ \\
\hline$M S F T$ & & 1 & $1.969+0.0079 t$ \\
\hline$A T \& T$ & & & 1 \\
\hline Medium Term & $I B N$ & $M S F$ & $A T \& T$ \\
\hline$I B M$ & {$\left[\begin{array}{ll}1 & 1 .\end{array}\right.$} & $76+0.0056 t$ & $2.797+0.089 t$ \\
\hline$M S F T$ & & 1 & $1.899+0.1125 t$ \\
\hline$A T \& T$ & L & & 1 \\
\hline Long Term & $I B M$ & $M S F T$ & $A T \& T$ \\
\hline$I B M$ & {$\left[\begin{array}{ll}1 & 1.7\end{array}\right.$} & $3-0.089 t$ & $2.943+0.0104 t]$ \\
\hline$M S F T$ & & 1 & $1.668+0.0175 t$ \\
\hline$A T \& T$ & & & 1 \\
\hline
\end{tabular}

Plots of estimated dynamic priorities versus actual are shown in Figure 13.

It may be possible to aggregate a judgment function into a number by assuming that each discrete judgment on a curve arises from different observations about a future determined not by a point but by an interval of time. In that case one uses the geometric mean of all these judgments leading to a product integral. As in the discrete case where we have for the product of the judgments $x_{1}, x_{2}, \ldots, x_{p}$ :

$$
x_{1} x_{2} \ldots x_{p}=e^{\log x_{1} x_{2} \ldots x_{p}}=\prod_{i=1}^{p} e^{\log x_{i}}=e^{\sum_{i=1}^{p} \log x_{i}} \rightarrow e^{\int \log x(\alpha) d \alpha}
$$

This product reduces to a product integral yielding a single number for an answer, for our case we replace $\alpha$ by $t$ and $x(\alpha)$ by $x(t)$ in the integral above to obtain a number for each judgment.

Finally, one can use the criterion $a_{i j} w_{j} / w_{i}>>1$ of the AHP or the gradient approach (more complex in the dynamic case) to improve overall consistency. One would possibly be dealing with nonlinear inequalities. 


\section{Estimated vs. Actual}

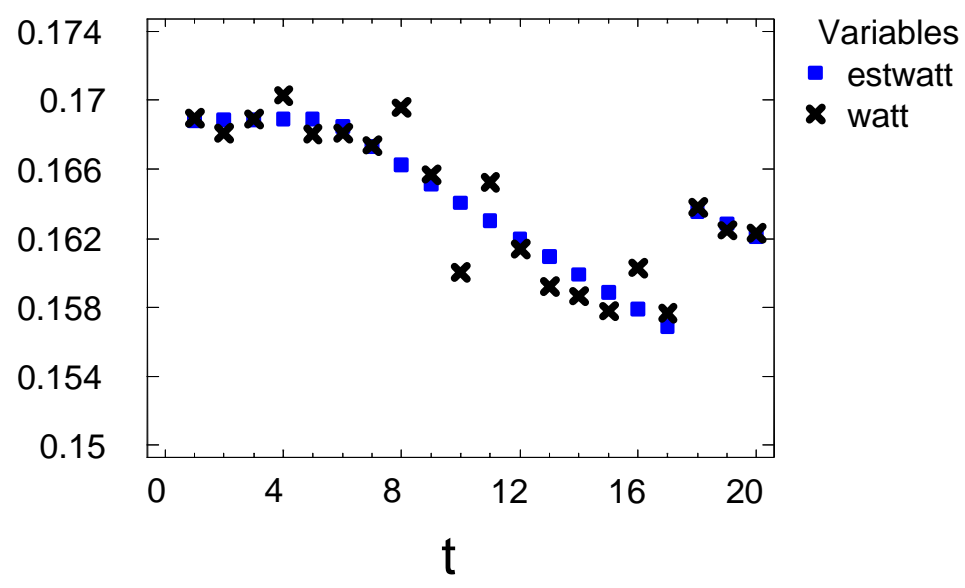

Estimated vs. Actual

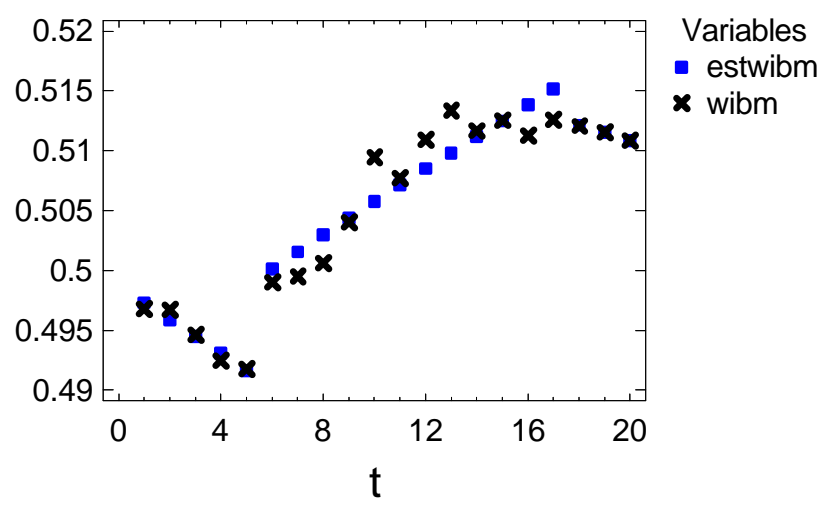

Estimated vs. Actual

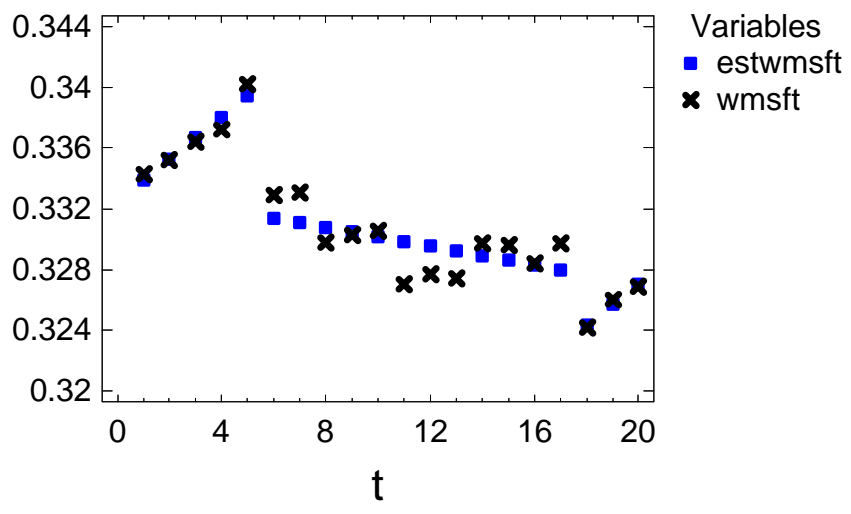

Figure 13 Estimated versus Actual Relative Values for the Three Companies 


\section{A Complete Example with Paired Comparisons}

Let us consider the case of tangibles. We have two people, the first is $a$ years older than the second, where $a=$ 30. As seen in Figure 14 as time lapses the difference between their ages remains constant. However, the ratio of their ages in the $(1,2)$ position in the paired comparison matrix below is a hyperbola given by $(a+t) / t=1+a / t$ as shown in Figure 15. Initially the ratio is infinite when $t=0$. As time lapses this ratio converges asymptotically to the line $x(t)=1$. The pairwise comparison matrix formed by taking the ratios of the functions of age of the two people is given by:

$$
\left[\begin{array}{cc}
1 & (a+t) / t \\
t /(a+t) & 1
\end{array}\right]
$$

which has the normalized principal right eigenvector :

$$
w_{1}(t)=(a+t) /(a+2 t), w_{2}(t)=t /(a+2 t) .
$$

The ideal mode solution is $1, t / t+a, t>0, a \geq 0$, with a graph comprised of a horizontal line and a curve as shown in Figure 16. Figure 17 is a plot of the normalized eigenvector components.

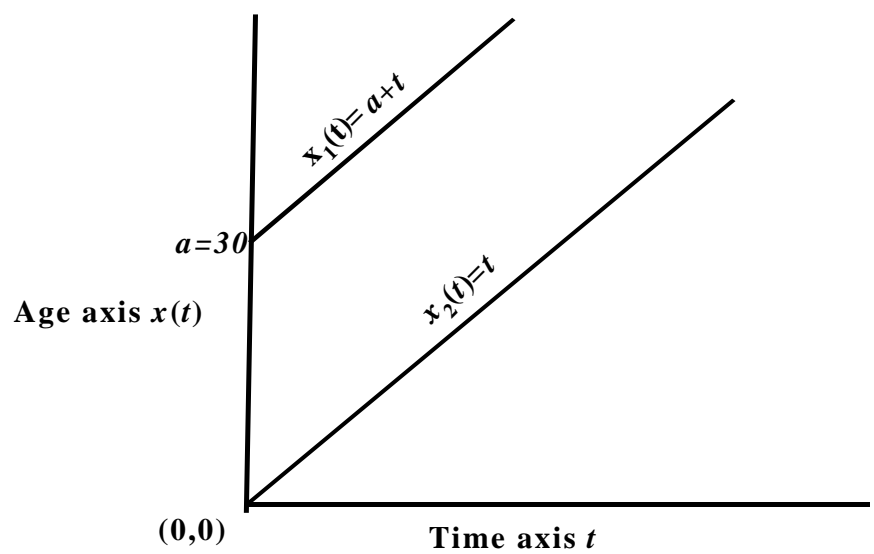

Figure 14 Age as a Function of Time 


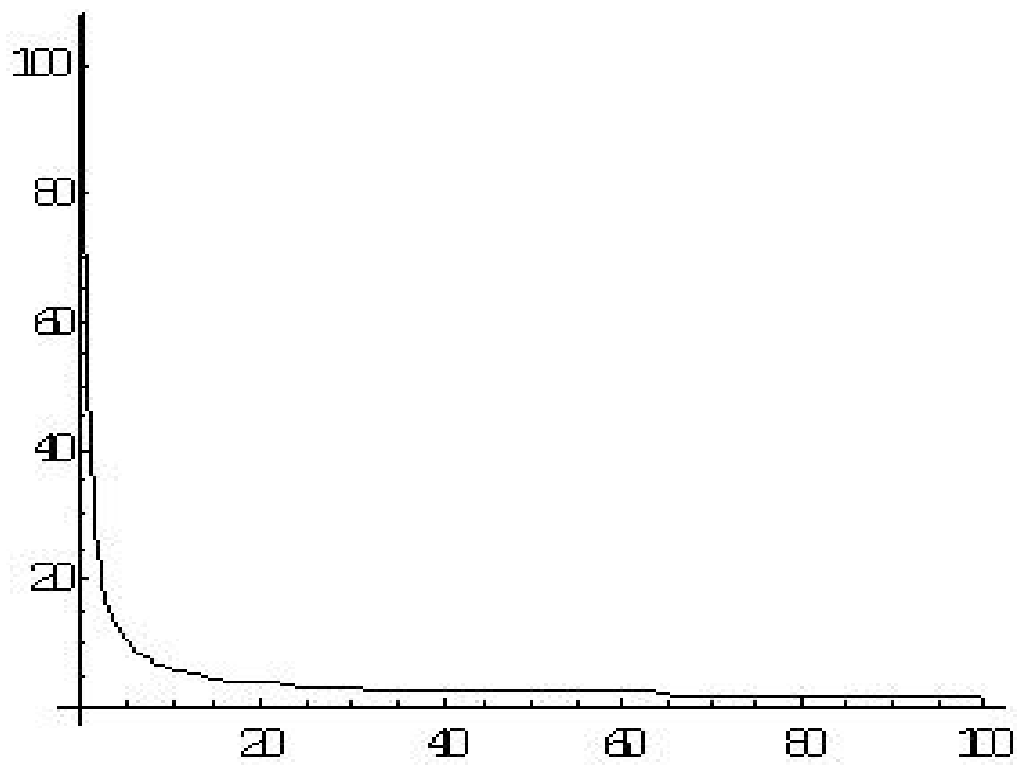

Figure 15 Hyperbola; $x_{1}(t) / x_{2}(t)=1+30 / t$

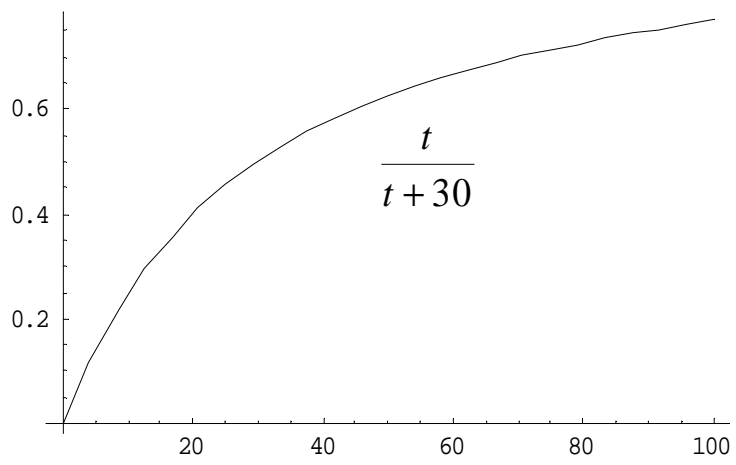

Figure 16 Graph of the Ratio of the Functions $x_{2}(t) / x_{1}(t)=t /(t+30)$ 


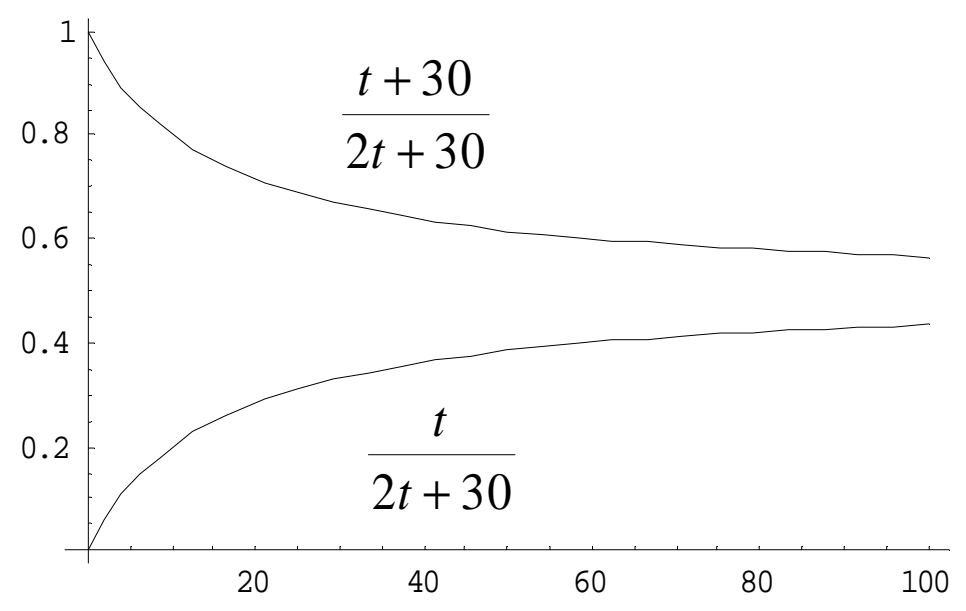

Figure 17 Graph of Normalized Time Dependent Eigenvector $w_{1}(t), w_{2}(t)$ with $\mathbf{a}=\mathbf{3 0}$

We continue our illustration of a multicriteria problem by considering another attribute besides age that is measurable over time, and that is strength. Let us assume that strength increases to a certain value $\leq 1$ and then decreases with age eventually to zero at death as in Figure 18. Assume that Figure 18 describes the strengths of the two individuals and that the corresponding equations are:

$$
y_{1}(t)=1-\frac{1}{2500}(t-20)^{2}, y_{2}(t)=1-\frac{1}{2500}(t-50)^{2}
$$

Forming the ratios of the functions that represent the relative strengths of the individuals we have the paired comparison matrix below:

$$
\left[\begin{array}{cc}
1 & \frac{y_{1}(t)}{y_{2}(t)} \\
\frac{y_{2}(t)}{y_{1}(t)} & 1
\end{array}\right]
$$

The above matrix has a normalized eigenvector of $\left[y_{1}(t), y_{2}(t)\right]$ which gives the solution vector $\left[u_{1}(t), u_{2}(t)\right]$ with:

$$
\begin{aligned}
& u_{1}(t)=1-\frac{1}{2500}(t-20)^{2} /\left[1-\frac{1}{2500}(t-20)^{2}+1-\frac{1}{2500}(t-50)^{2}\right] \\
& u_{2}(t)=1-\frac{1}{2500}(t-50)^{2} /\left[1-\frac{1}{2500}(t-20)^{2}+1-\frac{1}{2500}(t-50)^{2}\right]
\end{aligned}
$$

The graph of this time dependent eigenvector solution of strength is shown in Figure 19. 


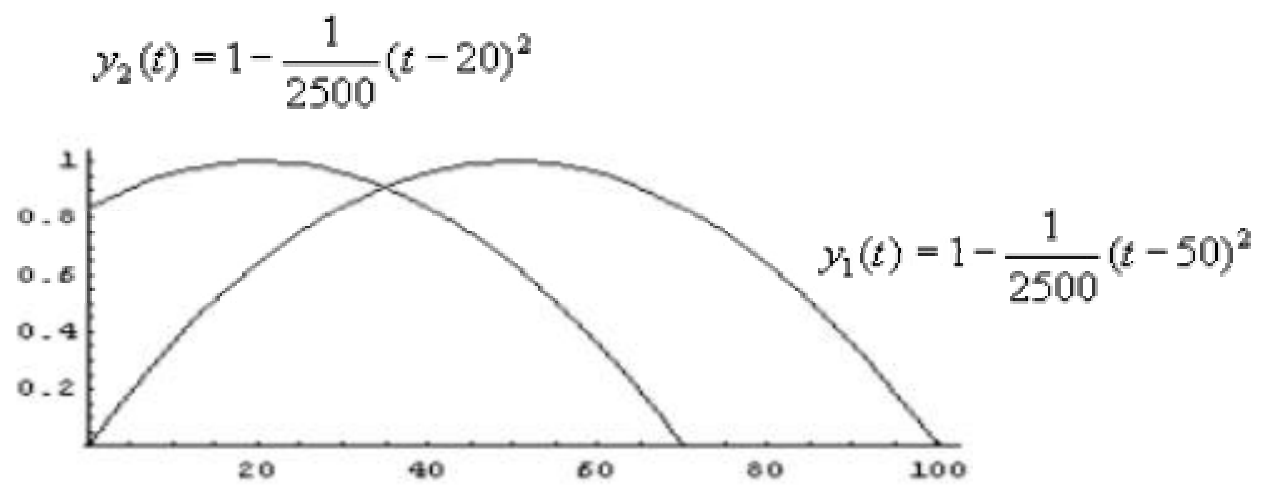

Figure 18 Graph of the Two Strength Functions $\left[y_{1}(t), y_{2}(t)\right]$

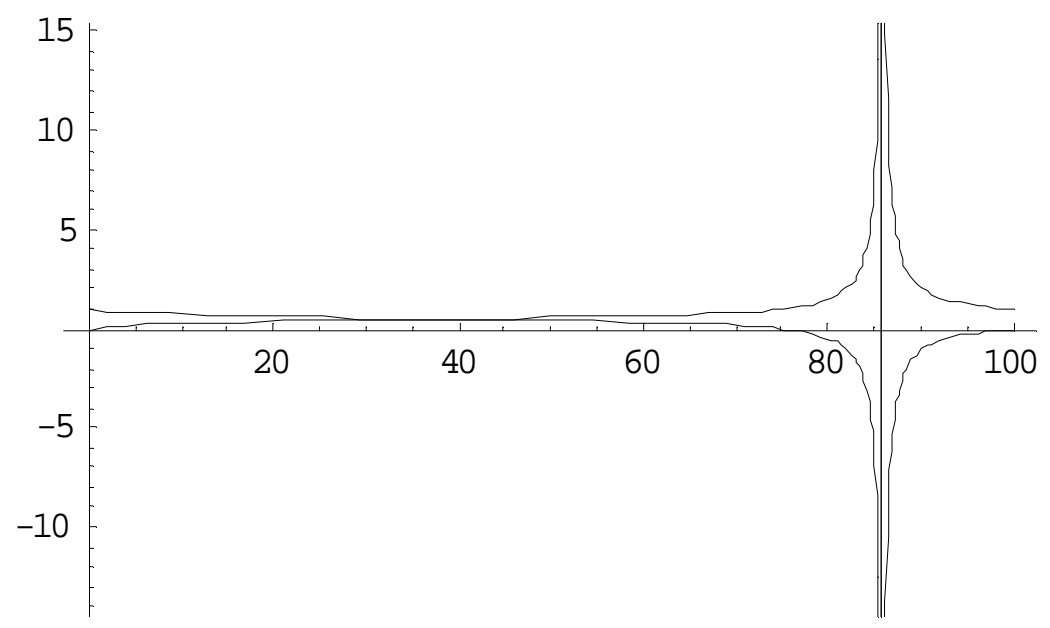

Figure 19 Time Dependent Eigenvector Solution of Strength Graph $\left[u_{1}(t), u_{2}(t)\right]$

We obtain the composite vector of the two alternatives, age and strength, by combining the two vectors obtained for age and strength.

Finally let us assume that the two criteria age and strength are equally important so we can weight and add the two vectors $w_{1}(t), w_{2}(t)$ with $a=30$ and $\left[u_{1}(t), u_{2}(t)\right]$. We might also have given the criteria time dependent weights to do the weighting and adding. We have the following solution for our final composite vector $\left[z_{1}(t), z_{2}(t)\right]$. Its graph is shown in Figure 20.

$z_{1}(t)=\left[\frac{1}{2}(30+t) /(30+2 t)\right]+\frac{1}{2}\left[1-\frac{1}{2500}(t-20)^{2}\right] /\left[1-\frac{1}{2500}(t-20)^{2}+1-\frac{1}{2500}(t-50)^{2}\right]$,

$z_{2}(t)=\left[\frac{1}{2} t /(30+2 t)\right]+\frac{1}{2}\left[1-\frac{1}{2500}(t-50)^{2}\right] /\left[1-\frac{1}{2500}(t-20)^{2}+1-\frac{1}{2500}(t-50)^{2}\right]$. 


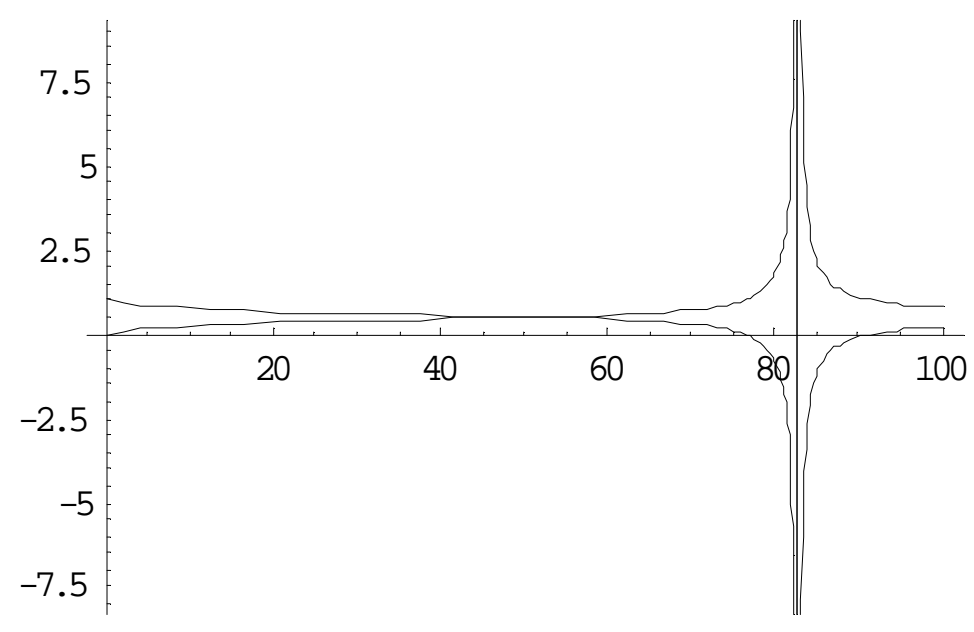

Figure 20 Graph of Synthesized Solution $\left[z_{1}(t), z_{2}(t)\right]$

\section{Hypermatrix of the Brain}

How do we maintain an ongoing record of the signals transmitted in the brain that is updated, revised, and synthesized, to capture the transient information that is communicated through neural firings? Synthesis in the brain is the most time dependent operation we know of intimately. The brain creates instantaneous impressions that are synthesized from one instant to another.

The approach we follow to represent interactions in the brain is a result of modeling the network of neurons by a graph, whose nodes are the neurons themselves, and whose synaptic connections with other neurons are arcs or line segments that connect the nodes. Electric potentials are measured across the arcs. We assign the potentials direction so that they are read in a positive direction. In the opposite direction we assign them a zero value.

Thus we represent the neurons of the brain conceptually by listing them in a column on the left of the hypermatrix (Table 7 and Figure 21) and listing them again in a row above the columns of the matrix. We assume that for convenience, we have arranged the neurons into components, which correspond to subcomponents or layers and these in turn according to the components or modules to which they belong. One can then enter a zero or a one in each column of the matrix to indicate whether a neuron in a row synapses with a neuron in a column. In fact, instead of the number one, a positive integer can be used to indicate the number of synapses that a neuron on the left has with a neuron at the top of the matrix. In that case each column of the matrix would represent the number of synapses of all neurons with a given neuron represented at the top of the matrix. That would be the most elementary way to represent the connections of the brain. It all hangs together in one piece, because every element is connected to some other element. Such a representation in a matrix can be modified by multiplying its nonnegative integer entries by the damped periodic oscillations of period one corresponding to the neuron with which the synapses are associated. In neural terminology, summing the elements in a column corresponds to spatial summation at an instant of time. The different components of the hypermatrix are represented as block supermatrices (Table 7). The control subsystems are connected to the supermatrices, which they control, and among themselves, and also to a higher-level of control components. 
Table 7 The Hypermatrix

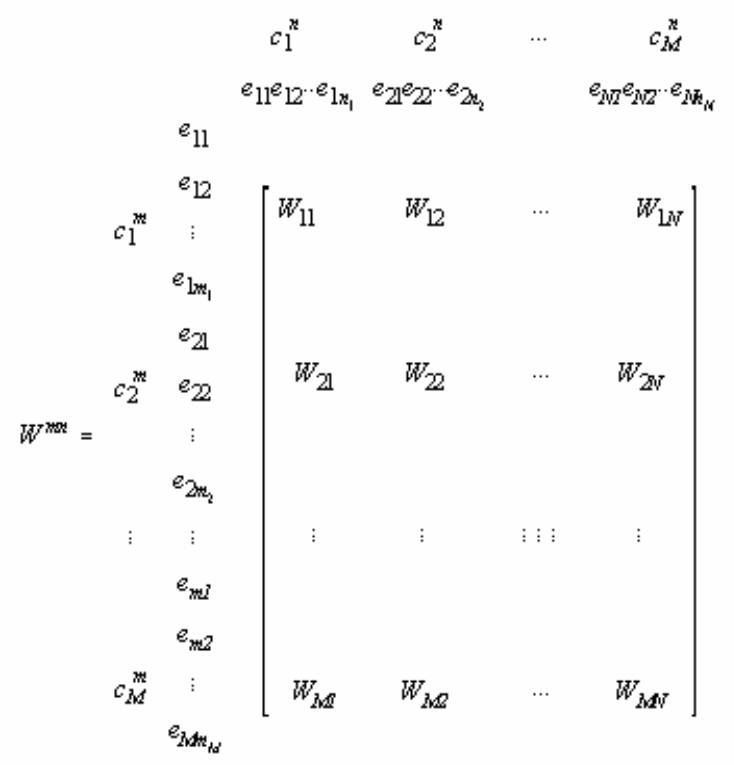

The $\mathrm{i}, \mathrm{j}$ block of this matrix is a supermatrix whose entries are matrices whose columns are principal right eigenfunctions. They are obtained as follows.

We offer the reader in Figures 21 and 22 a hard-worked-on but rudimentary application of the hypermatrix to modules and submodules of the brain to illustrate what we have in mind. We warn the reader that we are simply using imagination to brave the complexity. The size of the hypermatrix to describe the brain would be of the order of 100 billion by 100 billion (we have not consulted the Cray Research people about whether the development of their supercomputers comes close to satisfying the dicta of brain computing). It is far beyond our capability to handle the size of such a matrix, or know enough about the physical reality of the brain and its synapses to create the entries in the matrix. Figure 20 shows the supermatrix of vision as part of the hypermatrix.

\section{Synthesis}

The most significant observation about the brain, which consists of many individual neurons, is that it is a synthesizer of the firings of individual neurons into clusters of information and these in turn into larger clusters and so on, leading to an integrated whole.

Due to their sequential nature, the firings of a neuron that precede other neurons would be lost unless there is something like a field in which all the firings fit together to form a cohesive entity which carries information. Is there a field in the brain and where is it? We believe that the process of analytic continuation in the theory of functions of a complex variable provides insight into how neurons seem to know one another. On page 373, Kuffler and Nicholls in their often-cited book say, "The nervous system appears constructed as if each neuron had built into it an awareness of its proper place in the system." That is what analytic continuation does. It conditions neurons to fall on a unique path to continue information that connects with information processed by adjacent neurons with which it is connected. The uniqueness of analytic continuation has the striking consequence that something happening on a very small piece of a connected open set completely determines what is happening in the entire set, at great distances from the small piece.

By raising the hypermatrix to powers one obtains transitive interactions. This means that a neuron influences another neuron through intermediate neurons. All such two-step interactions are obtained by squaring the matrix. Three step interactions are obtained by cubing the matrix and so on. By raising the matrix to sufficiently large powers, the influence of each neuron on all the neurons with which one can trace a connection, yields the transient influence of neurons in the original hypermatrix Multiplying the hypermatrix by itself allows for combining the functions that represent the influence from pre-to post- synaptic neurons to 
accumulate all the transitive influences from one neuron to another and allow for feedback. The Fourier transform that takes place as a result of firing and the density of the resulting firings give us the desired synthesis. Depending on what part of the brain is operational and participating in the synthesis, different physical and behavioral attributes are observed to take place, including consciousness related to the Fourier transform of the single valued sensory functions.

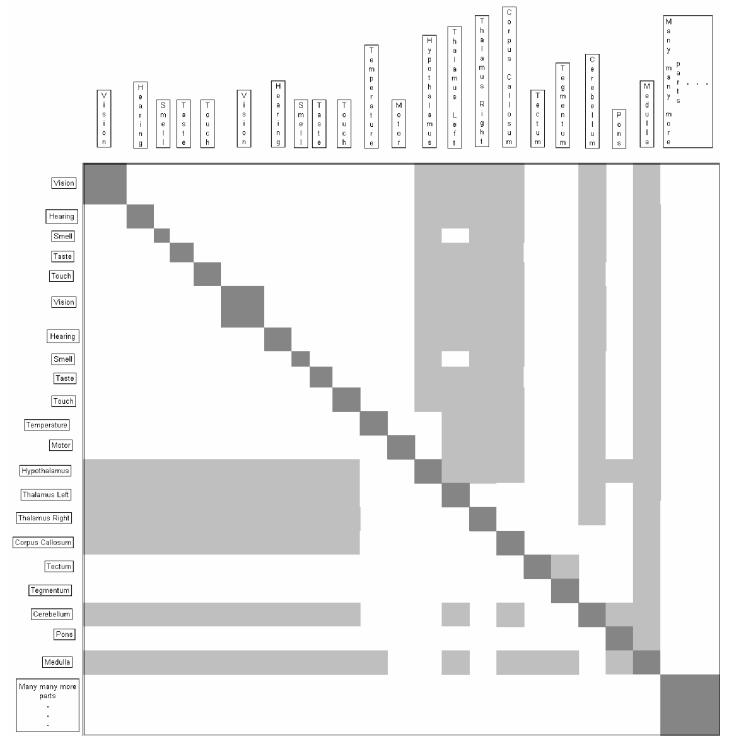

Figure 21 The Hypermatrix of the Brain

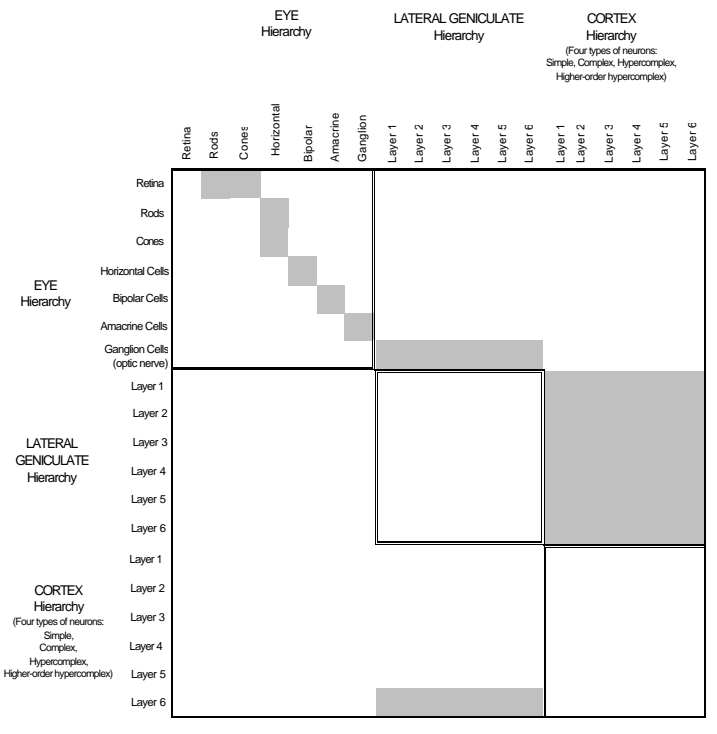

Figure 22 The Supermatrix of Vision

\section{Technical Discussion}

The foregoing discrete formulation (see my book: The Brain),

$$
\begin{gathered}
\sum_{j=1}^{n} a_{i j} w_{j}=\lambda_{\max } w_{i} \\
\sum_{i=1}^{n} w_{i}=1
\end{gathered}
$$

with $a_{j i}=1 / a_{i j}$ or $a_{i j} a_{j i}=1$ (the reciprocal property), $a_{i j}>0$ generalizes to the continuous case through Fredholm's integral equation of the second kind (which we can also derive directly from first principles to describe the response of a neuron to stimuli):

$$
\int_{a}^{b} K(s, t) w(t) d t=\lambda_{\max } w(s)
$$

where instead of the matrix $A$ we have a positive kernel, $K(s, t)>0$. A solution $w(s)$ of this equation is a right eigenfunction.

The standard way in which (3) is written is to move the eigenvalue to the left hand side which gives it the reciprocal form

$$
\lambda \int_{a}^{b} K(s, t) w(t) d t=w(s)
$$


with the normalization condition:

$$
\int_{a}^{b} w(s) d s=1
$$

An eigenfunction of this equation is determined to within a multiplicative constant. If $w(t)$ is an eigenfunction corresponding to the charateristic value $\lambda$ and if $C$ is an arbitrary constant, we see by substituting in the equation that $C w(t)$ is also an eigenfunction corresponding to the same $\lambda$. The value $\lambda=0$ is not a characteristic value because we have the corresponding solution $w(t)=0$ for every value of $t$, which is the trivial case, excluded in our discussion. Here also, we have the reciprocal property

$$
K(s, t) K(t, s)=1
$$

so that $K(s, t)$ is not only positive, but also reciprocal. An example of this type of kernel is $K(s, t)=\mathrm{e}^{\mathrm{s}-\mathrm{t}}=\mathrm{e}^{\mathrm{s}} / \mathrm{e}^{\mathrm{t}}$. As in the finite case, the kernel $K(s, t)$ is consistent if it satisfies the relation

$$
K(s, t) K(t, u)=K(s, u) \text {, for all } s, t \text {, and } u
$$

It follows by putting $s=t=u$, that $K(s, s)=1$ for all $s$ which is analogous to having ones down the diagonal of the matrix in the discrete case.

The most important part of what follows is the derivation of the fundamental equation, a functional equation whose solution is an eigenfunction of our basic Fredholm equation.

Theorem $1 \mathrm{~K}(\mathrm{~s}, \mathrm{t})$ is consistent if and only if it is separable of the form:

$$
K(s, t)=k(s) / k(t)
$$

Theorem 2 If $\mathrm{K}(\mathrm{s}, \mathrm{t})$ is consistent, the solution of (4) is given by

$$
w(s)=\frac{k(s)}{\int_{S} k(s) d s}
$$

We note that this formulation is general and applies to all situations where a continuous ratio scale is needed. It applies equally to the derivation or justification of ratio scales in the study of scientific phenomena. We now determine the form of $k(s)$ and also of $w(s)$.

In the discrete case, the normalized eigenvector was independent of whether all the elements of the pairwise comparison matrix $A$ are multiplied by the same constant $a$ or not, and thus we can replace $A$ by $a A$ and obtain the same eigenvector. Generalizing this result we have:

$$
K(a s, a \mathrm{t})=a K(s, t)=k(a s) / k(a t)=a k(s) / k(t) \quad(10)
$$

which means that $K$ is a homogeneous function of order one.

Theorem 3 A necessary and sufficient condition for $w(s)$ to be an eigenfunction solution of Fredholm's equation of the second kind, with a consistent kernel that is homogeneous of order one is that it satisfy the functional equation

$$
w(a s)=b w(s)
$$

where $b=\alpha a$. 
It is clear that whatever aspect of the real world we consider, sight, sound, touch, taste, smell, heat and cold, at each instant, their corresponding stimuli impact our senses numerous times. A stimulus $S$ of magnitude $s$, is received as a similarity transformation $a s, a>0$ referred to as a dilation of $s$. It is a stretching if $a>1$, and a contraction if $a<1$. When relating response to a dilated stimulus of magnitude $a s$ to response to an unaltered stimulus whose magnitude is $s$, we have the proportionality relation we just wrote down:

$$
\frac{w(a s)}{w(s)}=b
$$

We refer to equation (11) as: The Functional Equation of Ratio Scales. Because of its wider implications in science, we may call it: The Fundamental Equation of Proportionality and Order.

If we substitute $s=a^{u}$ in (11) we have:

$$
w\left(a^{u-1}\right)-b w\left(a^{u}\right)=0
$$

Again if we write,

$$
w\left(a^{u}\right)=b^{u} p(u)
$$

we get:

$$
p(\mathrm{u}+1)-p(\mathrm{u})=0
$$

which is a periodic function of period one in the variable $u$ (such as $\cos \mathrm{u} / 2 \pi$ ). Note that if $a$ and $s$ are real, then so is $u$ which may be negative even if $a$ and $s$ are both assumed to be positive.

If in the last equation $p(0)$ is not equal to 0 , we can introduce $C=p(0)$ and $P(u)=p(u) / C$, we have for the general response function $w(s)$ :

$$
w(s)=C e^{\log b \frac{\log s}{\log a} P\left(\frac{\log s}{\log a}\right)}
$$

where $P$ is also periodic of period 1 and $P(0)=1$. Note that $C>0$ only if $p(0)$ is positive. Otherwise, if $p(0)<0$, $C<0$.

Near zero, the exponential factor which is equal to $s^{\log b / \log a}$, "slims" $w(s)$ if $\log b / \log a>0$ and "spreads" $w(s)$ if $\log b / \log a<0$. Because $s$ is the magnitude of a stimulus and cannot be negative, we do not have a problem with complex variables here so long as both $a$ and $b$ are real and both positive. Our solution in the complex domain has the form:

$$
w(z)=z^{\ln b / \ln a} P(\ln z / \ln a)
$$

Here $P(u)$ with $u=\ln z / \ln a$, is an arbitrary multivalued periodic function in $\mathrm{u}$ of period 1 . Even without the multivaluedness of $P$, the function $w(z)$ could be multivalued because $\ln \mathrm{b} / \mathrm{ln}$ a is generally a complex number. If $P$ is single-valued and $\ln b / \ln a$ turns out to be an integer or a rational number, then $w(z)$ is a single-valued or finitely multivalued function, respectively. This generally multivalued solution is obtained in a way analogous to the real case. 


\section{This Solution Leads to the Weber-Fechner Law}

$$
n=\frac{\left(\log s_{n}-\log s_{0}\right)}{\log \alpha}
$$

Note in (12) that the periodic function $\mathrm{P}(\mathrm{u})$ is bounded and the negative exponential leads to an alternating series. Thus, to a first order approximation one obtains the Weber-Fechner law for response to a stimulus s: $\mathrm{A} \log \mathrm{s}+\mathrm{B}$. We assume that $\mathrm{B}=0$, and hence the response belongs to a ratio scale.

In 1846 Weber found, for example, that people while holding in their hand different weights, could distinguish between a weight of $20 \mathrm{~g}$ and a weight of $21 \mathrm{~g}$, but could not if the second weight is only $20.5 \mathrm{~g}$. On the other hand, while they could not distinguish between $40 \mathrm{~g}$ and $41 \mathrm{~g}$, they could between $40 \mathrm{~g}$ and $42 \mathrm{~g}$, and so on at higher levels. We need to increase a stimulus $s$ by a minimum amount $\Delta \Delta s$ to reach a point where our senses can first discriminate between $s$ and $s+\Delta s . \Delta s$ is called the just noticeable difference (jnd). The ratio $r=\Delta s / s$ does not depend on $s$. Weber's law states that change in sensation is noticed when the stimulus is increased by a constant percentage of the stimulus itself. This law holds in ranges where $\Delta s$ is small when compared with $s$, and hence in practice it fails to hold when s is either too small or too large. Aggregating or decomposing stimuli as needed into clusters or hierarchy levels is an effective way for extending the uses of this law.

In 1860 Fechner considered a sequence of just noticeable increasing stimuli. He denotes the first one by $\mathrm{s}_{0}$. The next just noticeable stimulus is given by

based on Weber's law.

$$
s_{1}=s_{1}+\Delta s_{0}=s_{0}+\frac{\Delta s_{0}}{s_{0}} s_{0}=s_{0}(1+r)
$$

Similarly

In general

$$
s_{2}=s_{1}+\Delta_{s_{1}}=s_{l}(1+r)=s_{0}(1+r)^{2} \equiv s_{0} \alpha^{2}
$$

$$
s_{n}=s_{n-} \alpha=s_{0} \alpha^{n}(n=0,1,2, \ldots)
$$

Thus stimuli of noticeable differences follow sequentially in a geometric progression. Fechner noted that the corresponding sensations should follow each other in an arithmetic sequence at the discrete points at which just noticeable differences occur. But the latter are obtained when we solve for $n$. We have

$$
n=\frac{\left(\log _{s_{n}}-\log _{s_{0}}\right)}{\log \alpha}
$$

and sensation is a linear function of the logarithm of the stimulus. Thus if $\mathrm{M}$ denotes the sensation and $\mathrm{s}$ the stimulus, the psychophysical law of Weber-Fechner is given by

$$
M=a \log s+b, \quad a \neq 0
$$

We assume that the stimuli arise in making pairwise comparisons of relatively comparable activities. We are interested in responses whose numerical values are in the form of ratios. Thus $b=0$, from which we must have $\log \mathrm{s}_{0}=0$ or $\mathrm{s}_{0}=1$, which is possible by calibrating a unit stimulus. Here the unit stimulus is $\mathrm{s}_{0}$. The next noticeable stimulus is $s_{l}=s_{0} \alpha=\alpha$ which yields the second noticeable response $a \log \alpha$. The 
third noticeable stimulus is $s_{2}=s_{0} \alpha^{2}$ which yields a response of $2 a \log \alpha$. Thus we have for the different responses:

$$
\mathrm{M}_{0}=a \log \mathrm{s}_{0}, \mathrm{M}_{1}=a \log \alpha, \mathrm{M}_{2}=2 a \log \alpha, \ldots, \mathrm{M}_{\mathrm{n}}=\mathrm{n} a \log \alpha .
$$

While the noticeable ratio stimulus increases geometrically, the response to that stimulus increases arithmetically. Note that $\mathrm{M}_{0}=0$ and there is no response. By dividing each $\mathrm{M}_{i}$ by $\mathrm{M}_{1}$ we obtain the sequence of absolute numbers $1,2,3, \ldots$ of the fundamental 1-9 scale. Paired comparisons are made by identifying the less dominant of two elements and using it as the unit of measurement. One then determines, using the scale 1-9 or its verbal equivalent, how many times more the dominant member of the pair is than this unit. In making paired comparisons, we use the nearest integer approximation from the scale, relying on the insensitivity of the eigenvector to small perturbations (discussed below). The reciprocal value is then automatically used for the comparison of the less dominant element with the more dominant one. Despite the foregoing derivation of the scale in the form of integers, someone might think that other scale values would be better, for example using 1.3 in the place of 2 . Imagine comparing the magnitude of two people with respect to the magnitude of one person and using 1.3 for how many there are instead of 2 .

Stimuli received by the brain from nature are transformed to chemical and electrical neural activities that result in summation and synthesis. This is transformed to awareness of nature by converting the electrical synthesis (vibrations caused by a pattern) to a space-time representation. The way the brain goes back and forth from a pattern of stimuli to its electro-chemical synthesis and then to a representation of its response to that spaciotemporal pattern is by applying the Fourier transform to the stimulus and the inverse Fourier transform to form its response. What we have been doing so far is concerned with the inverse Fourier transform. We now need to take its inverse to develop expressions for the response.

We now show that the space-time Fourier transform of (13) is a combination of Dirac distributions. Our solution of Fredholm's equation here is given as the Fourier transform,

$$
\left((\omega)=\int_{-\infty}^{+\infty} F(x) e^{-2 \pi i \omega x} d x=C e^{\beta \omega} P(\omega)\right.
$$

whose inverse transform is given by:

$$
(1 / 2 \pi) \log a \sum_{-\infty}^{\infty} a{ }_{n}\left[\frac{(2 \pi n+\theta(b)-x)}{(\log a|b|+(2 \pi n+\theta(b)-x)} i\right] \cdot \delta(2 \pi n+\theta(b)-x)
$$

where $\delta(2 \pi n+\theta(b)-x)$ is the Dirac delta function. This is supporting evidence in favor of our ratio scale model.

\section{The Formation of Images and Sounds with Dirac Distributions}

Complex valued functions cannot be drawn as one does ordinary functions of three real variables. The reason is that complex functions contain an imaginary part. Nevertheless, one can make a plot of the modulus or absolute value of such a function. The basic assumption we made to represent the response to a sequence of individual stimuli is that all the layers in a network of neurons are identical, and each stimulus value is represented by the firing of a neuron in each layer. A shortcoming of this representation is that it is not invariant with respect to the order in which the stimuli are fed into the network. It is known in the case of vision that the eyes do not scan pictures symmetrically if they are not symmetric, and hence our representation must satisfy some order invariant principle. Taking into account this principle would allow us to represent images independently of the form in which stimuli are input into the network. For example, we recognize an image even if it is subjected to a rotation, or to some sort of deformation. Thus, the 
invariance principle must include affine and similarity transformations. This invariance would allow the network to recognize images even when they are not identical to the ones from which it recorded a given concept, e.g., a bird. The next step would be to use the network representation given here with additional conditions to uniquely represent patterns from images, sounds and perhaps other sources of stimuli such as smell. Our representation focuses on the real part of the magnitude rather than the phase of the Fourier transform. Tests have been made to see the effect of phase and of magnitude on the outcome of a representation of a complex valued function. There is much more blurring due to change in magnitude than there is to change in phase. Thus we focus on representing responses in terms of Dirac functions, sums of such functions, and on approximations to them without regard to the coefficients in (15).

The functions result from modeling the neural firing as a pairwise comparison process in time. It is assumed that a neuron compares neurotransmitter-generated charges in increments of time. This leads to the continuous counterpart of a reciprocal matrix known as a reciprocal kernel. A reciprocal kernel $\boldsymbol{K}$ is an integral operator that satisfies the condition $\boldsymbol{K}(s, t) \boldsymbol{K}(t, s)=1$, for all $s$ and $t$. The response function $w(s)$ of the neuron in spontaneous activity results from solving the homogeneous equation (4). If

$$
\lim _{\xi \rightarrow 0} K(\xi s, \xi t)
$$

exists, where $\boldsymbol{K}$ is a compact integral operator defined on the space $\boldsymbol{L}_{2}[0, b]$ of Lebesgue square integrable functions. If the reciprocal kernel $K(s, t) \geq 0$, on $0 \leq s, t \leq b$, is Lebesgue square integrable and continuously differentiable, then

$$
w(t)=t^{\alpha} e^{g(t)} / \int_{0}^{b} t^{\alpha} e^{g(t)} d t
$$

satisfies (4) for some choice of $g(t)$. Because finite linear combinations of the functions $\left\{t^{\alpha} e^{-\beta t}, \alpha, \beta \geq 0\right\}$ are dense in the space of bounded continuous functions $\mathbf{C}[0, b]$ we can approximate $t^{\alpha} e^{g(t)}$ by linear combinations of $t^{\alpha} e^{-\beta t}$ and hence we substitute $g(t)=-\beta t, \beta \geq 0$, in the eigenfunction $w(t)$. The density of neural firing is not completely analogous to the density of the rational numbers in the real number system. The rationals are countably infinite, the number of neurons is finite but large. In speaking of density here we may think of making a sufficiently close approximation (within some prescribed bound rather than arbitrarily close).

We use the functions:

$$
\left\{t^{\alpha} e^{-\beta t}, \alpha, \beta \geq 0\right\}
$$

to represent images and sounds.

Before we describe how the network can be used to represent images and sounds, we summarize the mathematical model on which the neural density representation is based.

Neural responses are impulsive and hence the brain is a discrete firing system. It follows that the spontaneous activity of a neuron during a very short period of time in which the neuron fires is given by:

$$
w(t)=\sum_{k=1}^{R} \gamma_{k}\left(t-\tau_{k}\right)^{\alpha} e^{-\beta\left(t-\tau_{k}\right)}
$$

if the neuron fires at the random times $\tau_{k}, k=1,2, \ldots, R$. Empirical findings support the assumption that $R$ and the times $\tau_{k}, k=1,2, \ldots, R$ are probabilistic. However, the parameters $\alpha$ and $\beta$ vary from neuron to neuron, but are constant for the firings of each neuron. Non-spontaneous activity can be characterized as a perturbation of background activity. To derive the response function when neurons are stimulated from external sources, we 
consider an inhomogeneous equation to represent stimuli acting on the neuron in addition to existing spontaneous activity. Thus, we solve the inhomogenous Fredholm equation of the 2 nd kind given by:

$$
w(s)-\lambda_{0} \int_{0}^{b} K(s, t) w(t) d t=f(s)
$$

This equation has a solution in the Sobolev space

$$
W_{p}^{k}(\Omega)
$$

of distributions (in the sense of Schwartz) in $\mathbf{L}_{p}(W)$ whose derivatives of order $k$ also belong to the space $\mathbf{L}_{p}(W)$, where $W$ is an open subset of $\mathbf{R}^{n}$.

We created a 2-dimensional network of neurons consisting of layers. For illustrative purposes, we assume that there is one layer of neurons corresponding to each of the stimulus values. Thus, if the list of stimuli consists of $n$ numerical values, we created $n$ layers with a specific number of neurons in each layer. Under the assumption that each numerical stimulus is represented by the firing of one and only one neuron, each layer of the network must also consist of $n$ neurons with thresholds varying between the largest and the smallest values of the list of stimuli. We also assumed that the firing threshold of each neuron had the same width. Thus, if the perceptual range of a stimulus varies between two values $\theta_{1}$ and $\theta_{2}$, and each layer of the network has $n$ neurons, then a neuron in the ith position of the layer will fire if the stimulus value falls between

$\theta_{1}+(i-1) \frac{\theta_{2}-\theta_{1}}{n-1}$ and $\theta_{1}+i \frac{\theta_{2}-\theta_{1}}{n-1}$.

\section{Picture Experiment}

In the graphics experiment the bird and rose pictures required 124 and 248 data points, respectively, whereas the sound experiment required 1000 times more data points. Once the $(x, y)$ coordinates of the points were obtained, the $\mathrm{x}$-coordinate was used to represent time and the $y$-coordinate to represent response to a stimulus. The numerical values associated with the drawings in Figures 23 and 24 were tabulated and the numbers provided the input to the neurons in the networks built to represent the bird and the rose. This task is computationally demanding even for such simple geometric figures as the bird and the rose. For example, for the bird picture, the stimuli list consists of 124 values, and we would need $124^{2}=15376$ neurons, arranged in 124 layers of 124 neurons each The network and the data sampled to form the picture given in Figure 23, were used to create a $124 \times 124$ network of neurons consisting of 124 layers with 124 neurons in each layer. Each dot in the figures is generated by the firing of a neuron in response to a stimulus falling within the neuron's lower and upper thresholds.

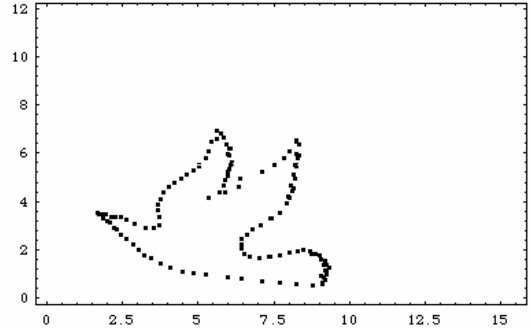

Figure 23 Bird

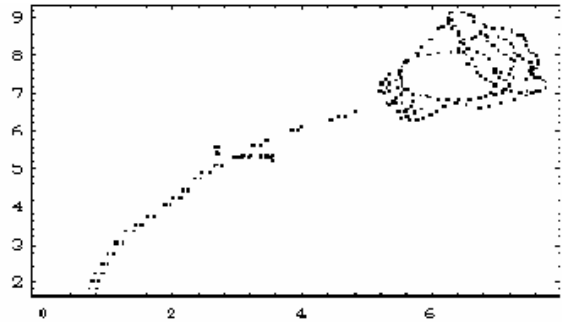

Fig 24 Rose

\section{Sound Experiment}

In the sound experiment we first recorded with the aid of Mathematica the first few seconds of Haydn's symphony no.102 in B-flat major and Mozart's symphony no. 40 in $\mathrm{G}$ minor. The result is a set of numerical amplitudes between -1 and 1 . Each of these amplitudes was used to make neurons fire when the amplitude falls within a prescribed threshold range. Under the assumption that each neuron fires in response to one stimulus, 
we would need the same number of neurons as the sample size, i.e., 117,247 in Haydn's symphony and 144,532 in Mozart's symphony. Our objective was to approximate the amplitude using one neuron for each amplitude value, and then use the resulting values in Mathematica to play back the music. A small sample of the numerical data for Mozart's symphony is displayed in Figure 25.

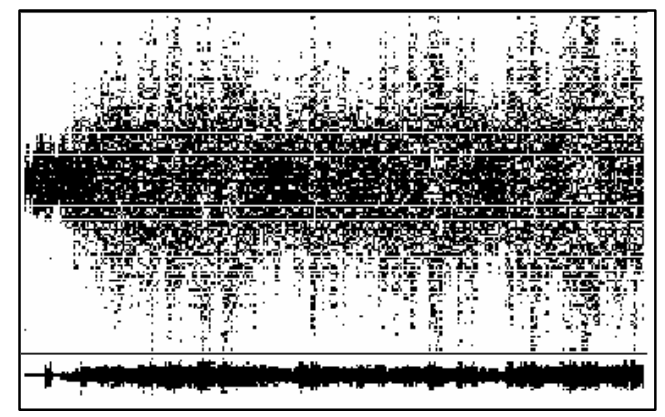

Figure 25 Mozart's Symphony No. 40

\section{Conclusions}

It appears that independently from whether people like to do or can do dynamic comparisons, there would always be technological problems with mathematical engineering design that require the use of AHP/ANP in dynamic form. The first applications may not be taxing to our ingenuity to deal with functions instead of numbers, but it is certain that soon after they will and I expect that extensions of this paper will make that appear even more plausible.

Perhaps one of the advantages of dynamic judgments may be that the requirement of homogeneity is given up to the mathematics of the functions one uses. It seems to me that while this may be reasonable for technical but not behavioral problems. One may be able to compare the relation between two machines constructed in a systematic way so that their relative behavior is predetermined. In that case homogeneity is not needed as a requirement on the fundamental scale.

One thing we have to learn to do is to create a scale that is truly associated with our intuition so that we can use it in the natural and spontaneous way people have learned to use the scale 1-9 that is associated with verbally expressed judgments. Intuitively I think this is a considerable worthy challenge of our effort to extend the AHP/ANP.

My appreciation goes to Luis G. Vargas for his dexterous and caring help with several tables and figures and for a part of our joint work on the brain.

\section{References}

Andreichicov, Alexander and Olga Andreichicova, "A Choice of a Perspective System for Vibration Isolation in Conditions of Varying Environment," pp. 13-24, ISAHP Proceedings 2001, Bern, Switzerland, 2001.

Blair, Andrew R., Robert Nachtmann, Thomas L. Saaty, Rozann Whitaker, "Forecasting the Resurgence of the U.S. Economy in 2001:An Expert Judgment Approach”, Socio-Economic Planning Sciences, 36, 77-91, 2002.

Horn, Roger.A, and Charles. R. Johnson, Matrix Analysis, Cambridge University Press, 1985.

King, R. Bruce, Beyond the Quartic Equation, Birkhaüser, 1994. 
Kuffler, S. and J.G. Nichols: From Neuron to rain. Sunderland, MA, USA: Sinauer Associates (1976).

Saaty, Thomas L., The Analytic Hierarchy Process, McGraw Hill International, New York, 1980. Translated to Russian, Portuguese, and Chinese. Revised edition published in paperback, RWS Publications, Pittsburgh, 1990, 1996.

Saaty, Thomas L., and Kevin Kearns, Analytical Planning, RWS Publications, 4922 Ellsworth Ave., Pittsburgh, PA 15213, 1991.

Saaty, Thomas L., Fundamentals of Decision Making with the Analytic Hierarchy Process, paperback, RWS Publications, 4922 Ellsworth Avenue, Pittsburgh, PA 15213-2807, original edition 1994, revised 2000.

Saaty, Thomas L., The Brain, Unraveling the Mystery of How it Works: The Neural Network Process, RWS Publications, 4922 Ellsworth Avenue, Pittsburgh, PA 15213, 2000

Saaty, Thomas L., The Analytic Network Process: Decision Making with Dependence and Feedback, RWS Publications, 4922 Ellsworth Ave., Pittsburgh, PA 15213, 1996, 2001 (completely revised).

Sturmfels, Bernd, "Solving algebraic equations in terms of A-hypergeometric series." Discrete Math, 210 (2000), No. 1-3, 171-181

Wind, Yoram, and T.L. Saaty, "Marketing Applications of the Analytic Hierarchy Process," Management Science, 26 (7), 1980.

\section{Historical Appendix}

Solution of the cubic and the quartic became common knowledge through the publication of the Ars Magna of Geronimo Cardano (1501-1576). But Cardano was not the original discoverer of the solution of either the cubic or the quartic. Generally the solution of the cubic is attributed to the Italian professor Scipione del Ferro (1465-1526), from Bologna, one of the oldest of the medieval universities and a school with a strong mathematical tradition.

The solution of the quartic is attributed to Ludovico Ferrari (1522-1565). The names of Niccolo Fontana (1500-1557) the stammerer (Tartaglia) is also associated with the solution of the cubic because he elaborated on Del Ferro's solution and became addicted to solving cubic equations. It was from Ferrari in 1547-48 that the history of this spectacular discovery became public knowledge. Ferrari reduced the solution of the general biquadratic equation to that of a cubic equation. It was Cardano who considered negative numbers and called them "fictitious".

Nearly 250 years later it became known form the works of Abel and Ruffini and from Galois theory that a quatric is the largest degree equation for which one can obtain the roots (eigenvalues in our problem) in the form of radicals. This means that the roots can be expressed as a finite formula involving only the four arithmetic operations and the extraction of roots. We have already given symbolic expressions for the principal eigenvalue and eigenvector in symbolic form for $n \leq 4$ in my first book on the AHP. Still even in these cases, it is a relatively complicated process to weight and synthesize time dependent priorities for the alternatives. Once we have the outcome, we can analyze its rate of change with respect to time by taking its derivative.

The mathematics to do this in general symbolic form is not here yet, nor is PC computer technology necessarily up to the task. To solve a quintic equation in symbolic form in order to get the principal eigenvalue we are told by the chart distributed in 1994 by Wolfram Research on the quintic equation that it requires a trillion bytes, or one gigabyte of storage. One wonders what solving higher order equations would require. The upshot is that we can do time dependent AHP/ANP numerically by simulation. One would express the judgments functionally but then derive the eigenvector from the judgments for a fixed moment of time, put the outcome in a 
supermatrix, solve the problem and derive the outcome for the alternatives. Repeating the process for different values of time generates a curve for the priorities. One then approximates these values by curves with a functional form for each coefficient.

\section{The Quintic and Higher Order Cases}

The poster distributed by Wolfram Research in 1994 gives a nice introduction to the classical methods for symbolic computation of the roots of an algebraic equation. All algebraic equations of degree 4 and less can be solved using only square and cubic roots. Solutions of algebraic equations using radicals are called radical solutions. Abel (1802-1829) proved that the general equation of degree higher than four could not be solved using radicals. Galois (1811-1832) provided a method to characterize equations that are solvable by radicals. His method involved the development of group theory to determine the effect of permuting the roots of the equation on functions of the roots. The solution of the quartic equation can be related to the $4 !=24$ symmetries of the tetrahedron, and those of the quintic equation to the $5 !=120$ symmetries of the icosahedron. The general quintic equation can be solved by Kiepert's algorithm (Auflðsung der Gleichungen vom Fünften Grades, J. für Math., 87, 114-133, 1878) using theta series (see below; power series, sometimes also called Taylor series have the form, $\sum_{n=0}^{\infty} c_{n} t^{n}$,Laurent series the form $\sum_{n=-\infty}^{\infty} c_{n} t^{n}$, and Puiseux series often encountered in this connection the form $\sum_{n=-\infty}^{\infty} c_{n} t^{n / k} \mathrm{k}$, a fixed natural number ). The fact that radicals cannot be used in all cases of the quintic and higher order equations leads to the question as to what type of functions are needed to solve equations of higher degree than 4. Hyperradicals is the term used for such functions. Hermite, for example, showed that elliptic modular functions provide solutions to the quintic equation. Tschirnhausen transformations are often used to simplify the form of an algebraic equation, solve it in the reduced form and then apply the inverse transformation to obtain the solution of the general equation. Actually, the equation $a_{0} x^{n}+a_{1} x^{n-1}+\ldots+a_{n-1} x+a_{n}=0, a_{0} \neq 0$ is reduced to $z^{n}+b_{1} z^{n-4}+\ldots+b_{n-1} x+b_{n}=0$ with three fewer terms, and thus the quintic equation takes the form $z^{5}+b_{4} z+b_{5}=0$. Theta functions are periodic functions that can be represented by series whose convergence is extraordinarily rapid. Jacobi and Abel in 1827 studied these functions for the first time. We have,

$$
\begin{aligned}
& \theta_{2}(z, q)=2 \sum_{n=0}^{\infty} q^{(n+1 / 2)^{2}} \cos (2 n+1) z \\
& \theta_{3}(z, q)=1+2 \sum_{n=0}^{\infty} q^{n^{2}} \cos 2 n z
\end{aligned}
$$

The elliptic modular function $\varphi(\mathrm{z})$ is given by:

$$
\varphi(z)=\sqrt[8]{\frac{\theta_{2}(0, z)^{4}}{\theta_{3}(0, z)^{4}}}
$$

Camille Jordan proved in 1870 that an algebraic equation of any degree can be solved in terms of modular functions. The following theorem of H. Umemura (Resolution of Algebraic Equations by theta Constants, in Tata Lectures on Theta II, D. Mumford, ed., pp. 3.261-3.272, Birkhaïser, Boston, 1984.) does not require the use of Tschirnhausen transformations. Let $f(x)=a_{0} x^{n}+a_{1} x^{n-1}+\ldots+a_{n}=0, a_{0} \neq 0$ be an algebraic equation irreducible over a subfield of the complex numbers. A root of this equation can be expressed in terms of theta functions of zero argument involving the period matrix derived from one of two types of hyperelliptic integrals. 